TRANSACTIONS OF THE

AMERICAN MATHEMATICAL SOCIETY

Volume 359, Number 6, June 2007, Pages 2851-2879

S 0002-9947(07)04152-9

Article electronically published on January 26, 2007

\title{
ASYMPTOTIC ESTIMATES OF MULTI-DIMENSIONAL STABLE DENSITIES AND THEIR APPLICATIONS
}

\author{
TOSHIRO WATANABE
}

Dedicated to Minoru Motoo on his 77th birthday

\begin{abstract}
The relation between the upper and lower asymptotic estimates of the density and the fractal dimensions on the sphere of the spectral measure for a multivariate stable distribution is discussed. In particular, the problem and the conjecture on the asymptotic estimates of multivariate stable densities in the work of Pruitt and Taylor in 1969 are solved. The proper asymptotic orders of the stable densities in the case where the spectral measure is absolutely continuous on the sphere, or discrete with the support being a finite set, or a mixture of such cases are obtained. Those results are applied to the moment of the last exit time from a ball and the Spitzer type limit theorem involving capacity for a multi-dimensional transient stable process.
\end{abstract}

\section{INTRODUCTION}

Since Lévy [20] in 1937, the study of stable processes and their distributions has been an important subject not only in theoretical probability but also in physics and finance. Many properties of univariate stable distributions are found in Zolotarev [43. However, there are few known results on the asymptotic properties of multivariate stable densities up to now. Only Pruitt and Taylor [28] investigated the asymptotic estimates of multivariate stable densities in a general setting. The others made certain strong assumptions on the spectral measure, for instance, symmetry, absolute continuity or discreteness. In this paper, surprisingly explicit estimates can be obtained for a multivariate $\alpha$-stable density $p(x)$ on $\mathbb{R}^{d}$. We first obtain the general upper and lower estimates without any technical assumption. See Theorem 1.1. In particular, we find the best possible and worst possible cases. Then we discuss the upper and lower asymptotic estimates of $p(x+y)$ as $|x| \rightarrow \infty$ with $y$ being bounded, from the point of view of their relation to the fractal dimensions of the spectral measure $\sigma$ on $S^{d-1}$. See Theorems 1.2 and 1.4. The asymptotic order delicately depends on the direction of $x /|x|$ in relation to the location of the support of $\sigma$. Thus we discover that a wide range of decay orders is possible. See Remark 1.3. Moreover, we solve the problem (Theorem 1.1) and the conjecture (Theorem 1.5) on the upper asymptotic estimates of multivariate stable densities in the classical work of Pruitt and Taylor 28] in 1969.

Received by the editors September 13, 2004 and, in revised form, June 13, 2005.

2000 Mathematics Subject Classification. Primary 60E07, 60G52; Secondary 60G51, 60J45.

Key words and phrases. Stable density, spectral measure, transient Lévy process, last exit time.

(C)2007 American Mathematical Society Reverts to public domain 28 years from publication 
In what follows, we use the terminology in Sato's book 32. Let $\left\{X_{t}: t \geqslant 0\right\}$ be a Lévy process on $\mathbb{R}^{d}, d \geqslant 1$, with generating triplet $(A, \nu, \gamma)$. Here $A$ is the Gaussian covariance matrix, $\nu$ is the Lévy measure, and $\gamma$ is the location parameter. That is,

$$
E \exp \left(i\left\langle z, X_{t}\right\rangle\right)=\exp (t \psi(z)), \quad z \in \mathbb{R}^{d}
$$

with

$$
\psi(z)=\int_{\mathbb{R}^{d}}\left(e^{i\langle z, x\rangle}-1-1_{\{|x| \leqslant 1\}}(x) i\langle z, x\rangle\right) \nu(d x)+i\langle\gamma, z\rangle-\frac{1}{2}\langle A z, z\rangle,
$$

where $\nu$ is a measure on $\mathbb{R}^{d}$ satisfying $\nu(\{0\})=0$ and $\int_{\mathbb{R}^{d}}\left(1 \wedge|x|^{2}\right) \nu(d x)<\infty$, $\gamma \in \mathbb{R}^{d}$, and $A$ is a nonnegative-definite matrix. If $A=0,\left\{X_{t}\right\}$ is said to be purely non-Gaussian. A Lévy process $\left\{X_{t}\right\}$ on $\mathbb{R}^{d}$ is called a trivial process if there is $c \in \mathbb{R}^{d}$ such that, for every $t, X_{t}=t c$ a.s.; otherwise $\left\{X_{t}\right\}$ is said to be nontrivial.

A set $B$ in $\mathbb{R}^{d}$ is called one-sided if there is $c \neq 0$ in $\mathbb{R}^{d}$ such that $B \subset\{x:\langle c, x\rangle \geqslant$ $0\}$. A measure $\rho$ on $\mathbb{R}^{d}$ is called one-sided if the support $S_{\rho}$ of $\rho$ is one-sided. A measure $\rho$ on $\mathbb{R}^{d}$ is called degenerate if there are $a \in \mathbb{R}^{d}$ and a proper linear subspace $V$ of $\mathbb{R}^{d}$ such that $S_{\rho} \subset a+V$; otherwise $\rho$ is called nondegenerate. A Lévy process $\left\{X_{t}\right\}$ on $\mathbb{R}^{d}$ is called degenerate if the distribution of $X_{t}$ is degenerate for every $t>0$ (equivalently, for some $t>0$ ); otherwise $\left\{X_{t}\right\}$ is called nondegenerate. See [32, Proposition 24.17 for conditions for nondegenerateness in terms of the generating triplet.

A Lévy process $\left\{X_{t}\right\}$ on $\mathbb{R}^{d}, d \geqslant 1$, is called stable if, for every $a>0$, there are $b>0$ and $c \in \mathbb{R}^{d}$ such that

$$
\left\{X_{a t}: t \geqslant 0\right\} \stackrel{\mathrm{d}}{=}\left\{b X_{t}+t c: t \geqslant 0\right\} .
$$

If $\left\{X_{t}\right\}$ is a nontrivial stable process, then define the index $\alpha$ of $\left\{X_{t}\right\}$ by $\alpha=$ $\log a / \log b$ for $a \neq 1$; this $\alpha$ is uniquely determined and $0<\alpha \leqslant 2$. In this case the process $\left\{X_{t}\right\}$ is sometimes called an $\alpha$-stable process. Let $\left\{X_{t}\right\}$ be a nontrivial stable process on $\mathbb{R}^{d}$. If $c=0$ in (1.3) for every $a>0$, we call $\left\{X_{t}\right\}$ a first-class stable process. Otherwise we call $\left\{X_{t}\right\}$ a second-class stable process. The process is first-class stable if and only if it is strictly stable in the terminology of [32; it is second-class stable if and only if it is stable but not strictly stable.

Let $\left\{X_{t}\right\}$ be a nontrivial $\alpha$-stable process on $\mathbb{R}^{d}$ with $\alpha \neq 2$. Then there is a probability measure $\sigma$ on $S^{d-1}=\left\{\xi \in \mathbb{R}^{d}:|\xi|=1\right\}$ such that

$$
\nu(B)=c \int_{S^{d-1}} \sigma(d \xi) \int_{0}^{\infty} 1_{B}(r \xi) r^{-1-\alpha} d r \quad \text { for } B \in \mathcal{B}_{\mathbb{R}^{d}},
$$

where $c$ is a positive constant independent of $\xi$ and $r$, and $\mathcal{B}_{\mathbb{R}^{d}}$ is the class of all Borel sets in $\mathbb{R}^{d}$. This $\sigma$ is uniquely determined and is called the spectral measure of the process $\left\{X_{t}\right\}$. We consider $\sigma$ as a measure on $\mathbb{R}^{d}$ with the understanding that $\sigma\left(\left(S^{d-1}\right)^{c}\right)=0$.

Let $\phi(r)$ be a positive and decreasing function on $(0, \infty)$. We use the words "increase" and "decrease" in the wide sense allowing flatness. Then we say $\phi \in \mathcal{D}$ if there is a constant $c_{1}>0$ such that $\phi(r) \leq c_{1} \phi(2 r)$ for $r>0$. A positive and decreasing function $\phi \in \mathcal{D}$ is called of dominated variation. Denote by $B_{a}$ an open ball in $\mathbb{R}^{d}$ with center 0 and radius $a$. Thus $\bar{B}_{a}=\{x:|x| \leqslant a\}$ and $B_{a}+y=\{x:|x-y|<a\}$. For the spectral measure $\sigma$ of an $\alpha$-stable process $\left\{X_{t}\right\}$ 
on $\mathbb{R}^{d}$ with $\alpha \neq 2$, define functions $\sigma_{\xi}(r)$ and $\sigma^{*}(r)$ for $r>0$ as

$$
\sigma_{\xi}(r)=\sigma\left(\xi+B_{1 / r}\right) \quad \text { and } \quad \sigma^{*}(r)=\sup _{\xi \in S^{d-1}} \sigma\left(\xi+B_{1 / r}\right) .
$$

Obviously $\sigma^{*}(\cdot)$ is positive and decreasing and $\sigma^{*}(\cdot) \in \mathcal{D}$. Thus there is a constant $b \geqslant 0$ such that

$$
\sigma^{*}(s r) \leqslant s^{-b} \sigma^{*}(r) \quad \text { for } 0<s<1 \text { and } r>0 .
$$

See Bingham et al. [3. Let $S_{\sigma}$ be the support of $\sigma$. Taking Carathéodory's theorem into account, we define

$$
\begin{aligned}
& C_{\sigma}^{0}(n)=\left\{\xi \in S^{d-1}: \xi=\sum_{j=1}^{n} c_{j} \xi_{j} \text { for some } c_{j}>0 \text { and } \xi_{j} \in S_{\sigma},\right. \\
&\left.j=1, \ldots, n, \text { such that } \xi_{1}, \ldots, \xi_{n} \text { are linearly independent }\right\}
\end{aligned}
$$

for $1 \leqslant n \leqslant d, T_{\sigma}(1)=C_{\sigma}^{0}(1)=S_{\sigma}$, and

$$
T_{\sigma}(n)=C_{\sigma}^{0}(n) \backslash \overline{C_{\sigma}^{0}(n-1)}
$$

for $2 \leqslant n \leqslant d$, where $\overline{C_{\sigma}^{0}(n-1)}$ is the closure of $C_{\sigma}^{0}(n-1)$. We have $\overline{C_{\sigma}^{0}(n)} \supset$ $\overline{C_{\sigma}^{0}(n-1)}$ for $2 \leqslant n \leqslant d$, since it is clear that $\overline{C_{\sigma}^{0}(n)} \supset C_{\sigma}^{0}(n-1)$. We write $C_{\sigma}^{0}=C_{\sigma}^{0}(d)$ and $C_{\sigma}=\overline{C_{\sigma}^{0}}$. Let int $C_{\sigma}$ be the interior of the set $C_{\sigma}$ in the relative topology on $S^{d-1}$. Note that in general, $C_{\sigma} \supset \bigcup_{n=1}^{d} T_{\sigma}(n)$ and int $C_{\sigma} \supset C_{\sigma}^{0}$. The set $C_{\sigma}^{0}$ is nonempty if $\left\{X_{t}\right\}$ is nondegenerate. These sets are introduced by Hiraba 12 , when $S_{\sigma}$ is a finite set.

In the rest of this section, we assume that $\left\{X_{t}\right\}$ is a nondegenerate $\alpha$-stable process on $\mathbb{R}^{d}$ with $0<\alpha<2$ and $d \geqslant 1$. Thus $\left\{X_{t}\right\}$ is purely non-Gaussian and $X_{t}$ has the probability density function $p(t, x)$ for $t>0$. We write $p(1, x)=p(x)$. We denote by $m$ the uniform probability measure on $S^{d-1}$. In the case where $0<\alpha<1$ and $\nu$ is one-sided, $p(x)$ can be zero, and thereby an additional condition $|x|>R$ with sufficiently large $R>0$ is needed in the lower estimates of $p(x)$. Otherwise, there is no need for that condition. Thus we promise to omit such a condition in the lower estimates of $p(x)$. First we give general asymptotic estimates applicable for all $\alpha$-stable densities.

Theorem 1.1. (i) There is a constant $c_{1}>0$ such that

$$
p(x) \leqslant c_{1}(1+|x|)^{-(1+\alpha)} \sigma^{*}(1+|x|) \quad \text { for } x \in \mathbb{R}^{d} .
$$

In particular, we have

$$
p(x) \leqslant c_{1}(1+|x|)^{-(1+\alpha)} \quad \text { for } x \in \mathbb{R}^{d} .
$$

(ii) Let $\xi^{0} \in S^{d-1}$. In the case where $0<\alpha<1$ and $\nu$ is one-sided, we make an additional assumption that $\xi^{0} \in \operatorname{int} C_{\sigma}$. (Otherwise we make no additional assumption.) Then for any $\delta_{1}>0$, there is $c_{2}>0$ such that it is independent of $\xi^{0}$ and

$$
p\left(r \xi^{0}+y\right) \geqslant c_{2}(1+r)^{-(1+\alpha)} \sigma_{\xi^{0}}(1+r) \quad \text { whenever }|y| \leqslant \delta_{1} .
$$

(iii) For any compact set $K_{1}$ in $C_{\sigma}^{0}$ and for any $\delta_{2}>0$, there is $c_{3}>0$ such that

$$
p(x+y) \geqslant c_{3}(1+|x|)^{-(1+\alpha) d} \quad \text { whenever } x /|x| \in K_{1},|y| \leqslant \delta_{2} .
$$

(iv) For any compact set $K_{2} \subset S^{d-1}$ with $K_{2} \cap C_{\sigma}=\emptyset$, for any $\delta_{3}>0$, and for any $c_{0}>0$, there is $c_{4}>0$ such that

$$
p(x+y) \leqslant c_{4} \exp \left(-c_{0}|x| \log |x|\right) \quad \text { whenever } x \neq 0, x /|x| \in K_{2},|y| \leqslant \delta_{3} \text {. }
$$


Pruitt and Taylor [28] showed the second assertion of (i) of Theorem 1.1 by a complicated Fourier-analytic method except in the case of second-class with $\alpha=1$; in this case the estimate (1.10) has been unproven up to now. Our method of proof is more probabilistic and simpler. Further Port 24] used (1.10) for the classification of transient stable processes into weakly transient and strongly transient. Thus he could not classify the case of second-class with $\alpha=1$ and $d=2$, which is shown to be strongly transient by Sato and Watanabe 34. Pruitt and Taylor 28 called the order $(1+|x|)^{-(1+\alpha)}$ of the density $p(x)$ the worst possible case. Thus the order $(1+|x|)^{-(1+\alpha) d}$ should be called the best possible case in the case where $C_{\sigma}^{0}=S^{d-1}$. The following theorem gives a proper asymptotic order of the density, which leads to a remark amazing even to the author.

Theorem 1.2. Let $\phi \in \mathcal{D}$ and let $K$ be a compact set in $S_{\sigma}$. Suppose that

$$
\limsup _{r \rightarrow \infty} \sup _{\xi \in S^{d-1}} \frac{\sigma\left(\xi+B_{1 / r}\right)}{\phi(r)}<\infty \text { and } \liminf _{r \rightarrow \infty} \inf _{\xi \in K} \frac{\sigma\left(\xi+B_{1 / r}\right)}{\phi(r)}>0 \text {. }
$$

In the case where $0<\alpha<1$ and $\nu$ is one-sided, we make an additional assumption that $K \subset \operatorname{int} C_{\sigma}$. Then given $\delta>0$, we can find $c_{1}>0$ and $c_{2}>0$ such that

$$
c_{1}(1+|x|)^{-(1+\alpha)} \phi(1+|x|) \leqslant p(x+y) \leqslant c_{2}(1+|x|)^{-(1+\alpha)} \phi(1+|x|)
$$

whenever $x /|x| \in K,|y| \leqslant \delta$.

Remark 1.3. Let $d \geqslant 2$ and fix $\alpha \in(0,2)$ in the above theorem. Let $\beta$ be an arbitrary real number in $(1+\alpha, d+\alpha)$. Then we can choose the spectral measure $\sigma$ in such a way that, for any $\delta>0$, there is a compact set $K$ in $S^{d-1}, c_{1}>0$, and $c_{2}>0$ such that

$$
c_{1}(1+|x|)^{-\beta} \leqslant p(x+y) \leqslant c_{2}(1+|x|)^{-\beta} \quad \text { whenever } x /|x| \in K,|y| \leqslant \delta .
$$

Indeed we can take $\sigma$ as a so-called $s$-measure on $S^{d-1}$ with $0<s<d-1$ such that $K=S_{\sigma}$ and $\beta=1+s+\alpha$ and that, for $c_{3}>0$ and $c_{4}>0$ independent of $x \in K$,

$$
c_{3} a^{s} \leqslant \sigma\left(K \cap\left(x+B_{a}\right)\right) \leqslant c_{4} a^{s} \quad \text { for } x \in K \text { and } a \in(0,1) .
$$

The existence of $s$-measure on $S^{d-1}$ is clear from that in $\mathbb{R}^{d-1}$.

Now we can give interesting asymptotic estimates of $p\left(r \xi^{0}+y\right)$ for $\xi^{0} \in T_{\sigma}(n)$ with $1 \leq n \leq d$ as $r \rightarrow \infty$ in relation to the fractal dimensions of the spectral measure $\sigma$. The upper estimate (1.19) and lower one (1.21) are attained in the cases of Theorems 3.8 and 3.10, respectively.

Theorem 1.4. Consider $\xi^{0} \in T_{\sigma}(n)$ with $1 \leq n \leq d$. Let $\phi_{1}, \phi_{2} \in \mathcal{D}$.

(i) Suppose that

$$
\limsup _{r \rightarrow \infty} \sup _{\xi \in S^{d-1}} \frac{\sigma\left(\xi+B_{1 / r}\right)}{\phi_{1}(r)}<\infty .
$$

Then, given $\delta_{1}>0$, we can find $c_{1}>0$ such that

$$
p\left(r \xi^{0}+y\right) \leqslant c_{1}(1+r)^{-(1+\alpha n)} \phi_{1}(1+r) \quad \text { whenever } r>0,|y| \leqslant \delta_{1} .
$$

(ii) Suppose that

$$
\liminf _{r \rightarrow \infty} \frac{\sigma\left(\xi+B_{1 / r}\right)}{\phi_{2}(r)}>0 \quad \text { for any } \xi \in S_{\sigma} .
$$


In the case where $0<\alpha<1$ and $\nu$ is one-sided, we make an additional assumption that $\xi^{0} \in \operatorname{int} C_{\sigma}$. Then, given $\delta_{2}>0$, we can find $c_{2}>0$ such that

$$
p\left(r \xi^{0}+y\right) \geqslant c_{2}\left((1+r)^{-(1+\alpha)} \phi_{2}(1+r)\right)^{n} \quad \text { whenever }|y| \leqslant \delta_{2} .
$$

Next, we give some new estimates of $p(x)$, which include the well-known rotationinvariant case. The upper estimate (1.23) was conjectured by Pruitt and Taylor [28] under the assumption (1.22). Dziubański [7] showed the existence of $\lim _{r \rightarrow \infty} r^{d+\alpha} p(r \xi)=k(\xi)$ with some $k(\xi)$ in some special case. Further related results were obtained by Arkhipov [1, Głowacki and Hebisch [10, and Głowacki 9]. They also obtained (1.23) under certain additional assumptions. Namely, [1] assumed first-class and smoothness of the density of the spectral measure. On the other hand, 7], 9], and [10] assumed symmetry. The results of those papers are interesting in view of ours.

Theorem 1.5. (i) If, for some $c_{1}>0$,

$$
\sigma(d \xi) \leqslant c_{1} m(d \xi) \quad \text { on } S^{d-1}
$$

then, for some $c_{2}>0$

$$
p(x) \leqslant c_{2}(1+|x|)^{-(d+\alpha)} \quad \text { for } x \in \mathbb{R}^{d} .
$$

The converse is also true except in the case where $0<\alpha<1$ and $\nu$ is one-sided.

(ii) Suppose that there is a nonempty compact set $K$ in $S^{d-1}$ such that

$$
\liminf _{r \rightarrow \infty} \inf _{\xi \in K} \frac{\sigma\left(\xi+B_{1 / r}\right)}{r^{-(d-1)}}>0 .
$$

In the case where $0<\alpha<1$ and $\nu$ is one-sided, we impose an additional condition that $K \subset \operatorname{int} C_{\sigma}$. Then, for any $\delta>0$, there is $c_{3}>0$ such that

$$
p(x+y) \geqslant c_{3}(1+|x|)^{-(d+\alpha)} \quad \text { whenever } x /|x| \in K,|y| \leqslant \delta .
$$

Remark 1.6. The condition (1.24) is satisfied if there are a nonempty relatively open set $U$ in $S^{d-1}$, a compact set $K$ in $\bar{U}$ and $c_{1}>0$ such that

$$
\sigma(d \xi) \geqslant c_{1} m(d \xi) \quad \text { on } U \quad \text { and } \quad \liminf _{a \rightarrow 0+} \inf _{\xi \in K} \frac{m\left(\left(\xi+B_{a}\right) \cap U\right)}{a^{d-1}}>0 .
$$

We prove the main results above in Section 3 after some preliminaries in Section 2. In Section 3, we add several other interesting results as follows. First we make a more precise observation of both the best possible and the worst possible cases. See Corollaries 3.4 and 3.5. Next we obtain the proper asymptotic order of the stable density $p(x+y)$ as $|x| \rightarrow \infty$ with $y$ being bounded for all or most of all the directions of $x /|x|$ in the case where the spectral measure $\sigma$ is absolutely continuous with respect to the uniform measure $m$ on $S^{d-1}$, or discrete with the support $S_{\sigma}$ being a finite set, or a mixture of those cases. See Theorems 3.8, 3.10 and 3.13. Then we apply those results to the moment of the last exit time from a ball and also to the Spitzer type limit theorems involving capacity in Section 4 for a second-class 
transient stable process with $d \geqslant 2$ and $1 \leqslant \alpha<2$. The reason why we study the order of $p(r \xi+y)$ not of $p(r \xi)$ for $\xi \in S^{d-1}$ is found in Lemma 4.5.

\section{Preliminaries}

In this section, we provide some preliminary results for Section 3. The support $S_{\rho}$ of a measure $\rho$ on $\mathbb{R}^{d}$ is the smallest closed set that carries the whole measure of $\rho$. We denote by $\rho^{n *} n$-th convolution power of the measure $\rho$. The distribution of an $\mathbb{R}^{d}$-valued random variable $X$ is denoted by $\mathcal{L}(X)$. We write $\left\{X_{t}\right\} \stackrel{\text { d }}{=}\left\{Y_{t}\right\}$ for two stochastic processes $\left\{X_{t}\right\}$ and $\left\{Y_{t}\right\}$ if they have an identical system of finitedimensional joint distributions. For a Lévy process $\left\{X_{t}\right\}$, we denote $\mu=\mathcal{L}\left(X_{1}\right)$ and $\mu^{t}=\mathcal{L}\left(X_{t}\right)$. The support of $\mu^{t}$ is denoted by $S\left(X_{t}\right)$. If $\mu^{t}$ is absolutely continuous with the density $p(t, x)$ being continuous in $x$, then the set $\{x: p(t, x)>0\}$ is denoted by $G\left(X_{t}\right)$.

Let $\left\{X_{t}\right\}$ be a nontrivial $\alpha$-stable process on $\mathbb{R}^{d}$ with $0<\alpha<2$ and Lévy measure $\nu$. Let $\psi(z)$ be the function defined in (1.2). If $0<\alpha<1$, then

$$
\psi(z)=\int_{\mathbb{R}^{d}}\left(e^{i\langle z, x\rangle}-1\right) \nu(d x)+i\left\langle\gamma_{0}, z\right\rangle
$$

where $\gamma_{0}$ is called the drift of $\left\{X_{t}\right\}$. If $1<\alpha<2$, then

$$
\psi(z)=\int_{\mathbb{R}^{d}}\left(e^{i\langle z, x\rangle}-1-i\langle z, x\rangle\right) \nu(d x)+i\left\langle\gamma_{1}, z\right\rangle
$$

where $\gamma_{1}$ is called the center of $\left\{X_{t}\right\}$ and $\gamma_{1}=E X_{1}$. We define $\tau \in \mathbb{R}^{d}$ as $\tau=\gamma_{0}$ for $0<\alpha<1, \tau=\gamma_{1}$ for $1<\alpha<2$, and $\tau=c \int_{S^{d-1}} \xi \sigma(d \xi)$ for $\alpha=1$. Here $c$ is the constant in (1.4). Note that the stable process $\left\{X_{t}\right\}$ is first-class if and only if $\tau=0$. If $\left\{X_{t}\right\}$ is nondegenerate, then $\mathcal{L}\left(X_{t}\right)$ has the probability density function $p(t, x)$. We write $p(1, x)=p(x)$. Then $p(x)$ is of class $C^{\infty}$ and satisfies the relation

$$
p(t, x)= \begin{cases}t^{-d / \alpha} p\left(t^{-1 / \alpha} x+\left(1-t^{1-1 / \alpha}\right) \tau\right) & (\alpha \neq 1), \\ t^{-d} p\left(t^{-1} x-(\log t) \tau\right) & (\alpha=1) .\end{cases}
$$

A Lévy process $\left\{X_{t}\right\}$ on $\mathbb{R}^{d}, d \geqslant 1$, is called semistable if, for some $a>0$ with $a \neq 1$, there are $b>0$ and $c \in \mathbb{R}^{d}$ satisfying (1.3). We call a semistable Lévy process, briefly, a semistable process. In this case we can choose $a>1$ without loss of generality. If $\left\{X_{t}\right\}$ is a nontrivial semistable process, then the index $\alpha$ can be defined in the same way as for nontrivial stable processes. See Sato [31, 32, concerning semistable processes.

A subset $H$ of $\mathbb{R}^{d}$ is said to be a closed additive semigroup if $H$ is a closed set such that $H+H \subset H$. If moreover $-H \subset H$, then $H$ is called a closed additive group. It should be noted that a closed additive semigroup $H$ in $\mathbb{R}^{d}$ is either onesided or a closed additive group. See Sato and Watanabe 33. Let $\left\{X_{t}\right\}$ be a nondegenerate $\alpha$-stable process on $\mathbb{R}^{d}$ with $0<\alpha \leqslant 2$ and Lévy measure $\nu$. The set $\operatorname{Sgp}(\nu)$ is the smallest closed additive semigroup containing $S_{\nu}$. We define a map $\Pi$ from $\mathbb{R}^{d} \backslash\{0\}$ to $S^{d-1}$ as $\Pi x=x /|x|$. Note that $C_{\sigma}=\Pi(\operatorname{Sgp}(\nu) \backslash\{0\})$. The following lemma is due to Taylor [38] and Port and Vitale [27. See Tortrat [39, Sharpe [35, and Sato and Watanabe 34] concerning the supports of general Lévy processes and semistable processes. 
Lemma 2.1. Let $\left\{X_{t}\right\}$ be a nondegenerate $\alpha$-stable process on $\mathbb{R}^{d}$ with $0<\alpha \leqslant 2$.

(i) Suppose that $0<\alpha<1$ and $\nu$ is one-sided. Then $\operatorname{Sgp}(\nu)$ is a one-sided, closed convex cone, and

$$
S\left(X_{t}\right)=t \gamma_{0}+\operatorname{Sgp}(\nu) \quad \text { and } \quad G\left(X_{t}\right)=t \gamma_{0}+\operatorname{int} \operatorname{Sgp}(\nu) \quad \text { for } t>0
$$

where int $\operatorname{Sgp}(\nu)$ is the interior of the set $\operatorname{Sgp}(\nu)$.

(ii) Suppose that $1 \leqslant \alpha \leqslant 2$, or suppose that $0<\alpha<1$ and $\nu$ is not one-sided. Then

$$
S\left(X_{t}\right)=G\left(X_{t}\right)=\mathbb{R}^{d} \text { for } t>0
$$

The following lemma was found by Taylor 38 .

Lemma 2.2. Let $\left\{X_{t}\right\}$ be a nondegenerate $\alpha$-stable process on $\mathbb{R}^{d}$ with $0<\alpha \leqslant 2$. Then the following conditions are equivalent:

$$
\begin{gathered}
0<\alpha<1 \text { and }-\gamma_{0} \notin \operatorname{int} \operatorname{Sgp}(\nu), \\
p(t, 0)=0 \text { for all } t>0, \\
p(t, 0)=0 \text { for some } t>0
\end{gathered}
$$

For the rest in this section let $\left\{X_{t}\right\}$ be a nondegenerate $\alpha$-stable process on $\mathbb{R}^{d}$ with $0<\alpha<2$. Our basic technique is to decompose $\left\{X_{t}\right\}$ into the sum of independent Lévy processes $\left\{Y_{t}\right\}$ and $\left\{Z_{t}\right\}$. That is, let $\left\{Y_{t}\right\}$ and $\left\{Z_{t}\right\}$ be independent Lévy processes such that $\left\{X_{t}\right\} \stackrel{\mathrm{d}}{=}\left\{Y_{t}+Z_{t}\right\}$ and $\left\{Z_{t}\right\}$ is a compound Poisson process with Lévy measure $\nu_{Z}$ equal to $\nu$ restricted to $\{|x|>\theta\}$ for some $\theta>0$. Thus the Lévy measure $\nu_{Y}$ of $\left\{Y_{t}\right\}$ is the restriction of $\nu$ to $\{|x| \leqslant \theta\}$. Let $\mathcal{L}\left(Y_{1}\right)=\mu_{Y}$ and $\mathcal{L}\left(Z_{1}\right)=\mu_{Z}$. Since $\left|\log \widehat{\mu}_{Z}(z)\right|$ is bounded, we get $\left|\widehat{\mu}_{Y}^{t}(z)\right| \leqslant e^{-c t|z|^{\alpha}+c_{1} t}$ with some $c>0$ and $c_{1}>0$ as in Proposition 24.20 of Sato [32. Thus $\mathcal{L}\left(Y_{t}\right), t>0$, has a density $p_{Y}(t, x)$, which is continuous in $(t, x)$, of class $C^{\infty}$ in $x$, and bounded for $(t, x) \in\left[\varepsilon_{1}, \varepsilon_{2}\right] \times \mathbb{R}^{d}$ for every $0<\varepsilon_{1}<\varepsilon_{2}<\infty$. We write $p_{Y}(t, x)=q(t, x)$ and $\mathcal{L}\left(Z_{t}\right)=\mu_{Z}^{t}=\lambda^{t}$. Further we write $q(x)=q(1, x)$ and $\lambda=\lambda^{1}$. As before, $B_{r}=\{x:|x|<r\}$; thus $\bar{B}_{r}=\{x:|x| \leqslant r\}$ and $B_{r}+y=\{x:|x-y|<r\}$. The following fact will be useful. See Sato and Watanabe [34] for the proof.

Lemma 2.3. Let $\left\{X_{t}\right\}$ be a nondegenerate $\alpha$-stable process on $\mathbb{R}^{d}, 0<\alpha<2$, with Lévy measure $\nu$. Then $S\left(Y_{t}\right)=S\left(X_{t}\right)$ and $G\left(Y_{t}\right)=G\left(X_{t}\right)$ for $t>0$.

Lemma 2.4. Let $c_{0}>0$. If $\theta$ in the above satisfies that $0<\theta<1 /\left(2 c_{0}\right)$, then there is $c_{1}$ such that

$$
q(x) \leqslant c_{1} \exp \left(-c_{0}|x| \log |x|\right) \quad \text { for }|x|>0 .
$$

Proof. Theorem 26.1 of 32 tells us that

$$
\int_{|y|>r} q(1 / 2, y) d y \leqslant c_{3} \exp \left(-2\left(c_{0}+\delta\right) r \log r\right) \quad \text { for } r>0
$$


with some positive constants $c_{3}$ and $\delta$. Let $c_{2}=\sup _{x \in \mathbb{R}^{d}} q(1 / 2, x)$. Now,

$$
\begin{aligned}
q(x) & =\int_{|x-y| \leqslant|x| / 2} q(1 / 2, x-y) q(1 / 2, y) d y+\int_{|x-y|>|x| / 2} q(1 / 2, x-y) q(1 / 2, y) d y \\
& \leqslant 2 c_{2} \int_{|y| \geqslant|x| / 2} q(1 / 2, y) d y \\
& \leqslant 2 c_{2} c_{3} \exp \left(-\left(c_{0}+\delta\right)|x| \log (|x| / 2)\right) \\
& \leqslant c_{4} \exp \left(-c_{0}|x| \log |x|\right)
\end{aligned}
$$

for large $|x|$ with some positive constant $c_{4}$. This gives (2.9).

The following simple lemma, together with Lemma 3.1, will be a key to prove the main results. All the estimates below are carried over under the condition that $\theta$ is given and fixed.

Lemma 2.5. Let $\varphi_{1}$ and $\varphi_{2}$ be nonnegative decreasing functions on $(0, \infty)$ with $\varphi_{1} \in \mathcal{D}$ and let $K_{1}$ and $K_{2}$ be compact sets in $S^{d-1}$.

(i) Asssume that there are $c_{1}>0$ and $\delta_{0}>0$ such that if $x \neq 0$ and $z$ satisfy $x /|x| \in K_{1}$ and $|z| \leqslant \delta_{0}|x|$, then

$$
\lambda\left(x+z+B_{1}\right) \leqslant c_{1} \varphi_{1}(1+|x|) .
$$

Then, for any $\delta_{1}>0$, there is $c_{2}>0$ such that if $x \neq 0$ and $y$ satisfy $x /|x| \in K_{1}$ and $|y| \leqslant \delta_{1}$, then

$$
p(x+y) \leqslant c_{2} \varphi_{1}(1+|x|) .
$$

(ii) In the case where $0<\alpha<1$ and $\nu$ is one-sided, we impose an additional condition that $K_{2} \subset \operatorname{int} C_{\sigma}$. Otherwise we impose no additional condition on $K_{2}$. Assume that there are $a>0, c_{3}>0$ and $R_{1}>0$ such that if $x /|x| \in K_{2}$ and $|x| \geqslant R_{1}$, then

$$
\lambda\left(x+B_{a}\right) \geqslant c_{3} \varphi_{2}(1+|x|) .
$$

Then, for any $\delta_{2}>0$, there are $c_{4}>0$ and $R_{2}>0$ such that if $x /|x| \in K_{2}$, $|x| \geqslant R_{2}$, and $|y| \leqslant \delta_{2}$, then

$$
p(x+y) \geqslant c_{4} \varphi_{2}(1+|x|) .
$$

Proof. (i) Since $p(t, x)=\int q(t, x-z) \lambda^{t}(d z)$, we have

$$
\begin{aligned}
p(x+y) & =\int q(-z) \lambda(x+y+d z) \\
& =\int_{|z| \leqslant \delta_{0}|x| / 2} q(-z) \lambda(x+y+d z)+\int_{|z|>\delta_{0}|x| / 2} q(-z) \lambda(x+y+d z) .
\end{aligned}
$$

Let $J_{1}$ and $J_{2}$ be the first and the second term in the last expression. We can choose $x_{j}, j=1, \ldots, N=N(x)$, with $\left|x_{j}\right| \leqslant \delta_{0}|x| / 2$ such that $\left\{z:|z| \leqslant \delta_{0}|x| / 2\right\} \subset$ $\bigcup_{j=1}^{N}\left(B_{1}+x_{j}\right)$, and there is $c_{5}>0$ independent of $N$ and $x$ satisfying $\sum_{j=1}^{N} Q_{j} \leqslant c_{5}$ for $Q_{j}=\sup _{z \in B_{1}+x_{j}} q(-z)$. This is possible by Lemma 2.4. Let $R=2 \delta_{1} / \delta_{0}$. If $x /|x| \in K_{1},|x| \geqslant R$, and $|y| \leqslant \delta_{1}$, then

$$
\begin{gathered}
\left|y+x_{j}\right| \leqslant|y|+\left|x_{j}\right| \leqslant(|y| /|x|)|x|+\left(\delta_{0} / 2\right)|x| \leqslant \delta_{0}|x|, \\
\lambda\left(x+y+x_{j}+B_{1}\right) \leqslant c_{1} \varphi_{1}(1+|x|)
\end{gathered}
$$


by (2.10). Hence

$$
J_{1} \leqslant \sum_{j=1}^{N} Q_{j} \lambda\left(x+y+x_{j}+B_{1}\right) \leqslant c_{1} c_{5} \varphi_{1}(1+|x|) .
$$

On the other hand, by Lemma 2.4 and $\varphi_{1} \in \mathcal{D}$,

$$
J_{2} \leqslant \sup _{|z|>\delta_{0}|x| / 2} q(-z) \leqslant c_{6} \exp \left(-c_{0}\left(\delta_{0}|x| / 2\right) \log \left(\delta_{0}|x| / 2\right)\right) \leqslant c_{7} \varphi_{1}(1+|x|)
$$

with some $c_{0}, c_{6}$, and $c_{7}$. Thus we get (2.11) under the condition $|x| \geqslant R$. Hence it holds without this condition with a suitable choice of $c_{2}$.

(ii) First, suppose that $1 \leqslant \alpha<2$, or suppose that $0<\alpha<1$ and $\nu$ is not one-sided. We have

$$
p(x+y) \geqslant \int_{B_{a}} q(y-z) \lambda(x+d z) .
$$

Let $c_{8}=\inf _{|y| \leqslant \delta_{2},|z|<a} q(y-z)$. Then $c_{8}>0$ by Lemma 2.1. Lemma 2.3, and the continuity of $q(\cdot)$. Thus, if $x /|x| \in K_{2},|x| \geqslant R_{1}$, and $|y| \leqslant \delta_{2}$, then

$$
p(x+y) \geqslant c_{8} \lambda\left(x+B_{a}\right) \geqslant c_{8} c_{3} \varphi_{2}(1+|x|) .
$$

Next, suppose that $0<\alpha<1, \nu$ is one-sided and $K_{2} \subset \operatorname{int} C_{\sigma}$. Recall that $q(z)>0$ for $z \in \gamma_{0}+\operatorname{int} \operatorname{Sgp}(\nu)$ (Lemmas 2.1 and 2.3) and that

$$
\operatorname{Sgp}(\nu)=\Pi^{-1} C_{\sigma} \cup\{0\}
$$

(the closedness of $\operatorname{Sgp}(\nu)$ under multiplication by positive reals in Lemma 2.1). Choose $r_{0}>0$ so large that $K^{\prime}=r_{0} K_{2}+\bar{B}_{a+\delta_{2}+\left|\gamma_{0}\right|} \subset \operatorname{int} \operatorname{Sgp}(\nu)$. Let $c_{9}=$ $\inf _{z \in \gamma_{0}+K^{\prime}} q(z)$. Then $c_{9}>0$. If $x /|x| \in K_{2},|x| \geqslant R_{1}+r_{0}$, and $|y| \leqslant \delta_{2}$, then

$$
\begin{aligned}
p(x+y) & \geqslant \int_{z \in-r_{0} K_{2}+B_{a}} q(y-z) \lambda(x+d z) \geqslant c_{9} \lambda\left(x-r_{0} K_{2}+B_{a}\right) \\
& \geqslant c_{9} \lambda\left(x-r_{0} x /|x|+B_{a}\right)=c_{9} \lambda\left(\left(|x|-r_{0}\right) x /|x|+B_{a}\right) \\
& \geqslant c_{9} c_{3} \varphi_{2}\left(1+\left(|x|-r_{0}\right)\right) \geqslant c_{10} \varphi_{2}(1+|x|)
\end{aligned}
$$

with some $c_{10}$.

Remark 2.6. Picard [22] studied the density estimates in small time for jump processes. In the case of first-class stable processes, his results are essentially similar to the statements of Lemma 2.5 because of the relation (2.3) with $\tau=0$. However, the arguments in Lemma 2.7 below are not therein, and thereby the relation to the spectral measure is not discussed in [22].

Let $l$ be a positive integer. For $\xi^{0}, \xi_{1}, \ldots, \xi_{l} \in S^{d-1}$ (not necessarily distinct), $r>0, y \in \mathbb{R}^{d}$, let

$$
I=I\left(\xi_{1}, \ldots, \xi_{l} ; r, \xi^{0}, y\right)=\int_{(\theta, \infty)^{l}} 1_{r \xi^{0}+y+B_{1}}\left(\sum_{j=1}^{l} r_{j} \xi_{j}\right) \prod_{j=1}^{l} \frac{d r_{j}}{r_{j}^{1+\alpha}}
$$

and

$$
J=J\left(l, r, \xi^{0}, y\right)=\int_{\left(S^{d-1}\right)^{l}} I\left(\xi_{1}, \ldots, \xi_{l} ; r, \xi^{0}, y\right) \prod_{j=1}^{l} \sigma\left(d \xi_{j}\right) .
$$


Note that $\nu_{Z}^{l *}\left(r \xi^{0}+y+B_{1}\right)=c^{l} J\left(l, r, \xi^{0}, y\right)$ with $c$ being the constant in (1.4). Denote the smallest linear subspace containing $\xi_{1}, \ldots, \xi_{l}$ by $\operatorname{Span}\left\{\xi_{1}, \ldots, \xi_{l}\right\}$. Define the set $\Xi_{n}$ with $1 \leq n \leq d$ as

$$
\begin{aligned}
\Xi_{n}= & \left\{\left(\xi_{1}, \ldots, \xi_{n}\right): \xi_{j} \in S^{d-1},\right. \\
& \left.j=1, \ldots, n, \text { such that } \xi_{1}, \ldots, \xi_{n} \text { are linearly independent }\right\} .
\end{aligned}
$$

For $\left(\xi_{1}, \ldots, \xi_{n}\right) \in \Xi_{n}$, let $P$ be a isometric linear mapping from $\operatorname{Span}\left\{\xi_{1}, \ldots, \xi_{n}\right\}$ to $\mathbb{R}^{n}$ and define $D\left(\xi_{1}, \ldots, \xi_{n}\right)=\left|\operatorname{det}\left(P \xi_{1}, \ldots, P \xi_{n}\right)\right|$ where $\operatorname{det}\left(P \xi_{1}, \ldots, P \xi_{n}\right)$ is the determinant considering each $P \xi_{j}$ as a column vector in $\mathbb{R}^{n}$. Obviously $D\left(\xi_{1}, \ldots, \xi_{n}\right)$ does not depend on the choice of the mapping $P$. Define

$$
A_{n}=\int_{\Xi_{n}} \frac{1}{D\left(\xi_{1}, \ldots, \xi_{n}\right)} \prod_{j=1}^{n} \sigma\left(d \xi_{j}\right) .
$$

Next we give some upper estimates of the integral $J$ for $\xi^{0} \in T_{\sigma}(n)$ with $1 \leqslant n \leqslant d$.

Lemma 2.7. Given $\xi^{0} \in T_{\sigma}(n)$ with $1 \leqslant n \leqslant d$, we can find $0<\delta<1$ and $R>0$ with the following properties:

(i) If $\xi_{1}, \ldots, \xi_{l} \in S_{\sigma}$ and $\operatorname{dim} \operatorname{Span}\left\{\xi_{1}, \ldots, \xi_{l}\right\} \leqslant n-1$, then $I=0$ whenever $|y| \leqslant \delta r$ and $r \geqslant R$.

(ii) There are constants $b \geqslant 0, c_{1}>0$ and $c_{2}>0$ determined by $\alpha, \theta, \delta$, and $n$, such that, if $\xi_{1}, \ldots, \xi_{l} \in S_{\sigma}$ and $\operatorname{dim} \operatorname{Span}\left\{\xi_{1}, \ldots, \xi_{l}\right\} \geqslant n$, then

$$
J \leqslant c_{1}\left(\begin{array}{l}
l \\
n
\end{array}\right) l^{(1+\alpha) n} \alpha^{-l} \theta^{-\alpha l} r^{-(1+\alpha) n} A_{n} \quad \text { whenever }|y| \leqslant \delta r / 2 \text { and } r \geqslant R
$$

and

$$
J \leqslant c_{2}\left(\begin{array}{l}
l \\
n
\end{array}\right) l^{\alpha n+b} \alpha^{-l} \theta^{-\alpha l} r^{-(1+\alpha n)} \sigma^{*}(r) \quad \text { whenever }|y| \leqslant \delta r / 2 \text { and } r \geqslant R .
$$

Proof. Given $\xi^{0}$ as above, choose $\delta>0$ so small and $R$ so large that, if $|y| \leqslant \delta r$ and $r \geqslant R$, then $\left(r \xi^{0}+y+B_{1}\right) \cap \Pi^{-1} \overline{C_{\sigma}^{0}(n-1)}=\emptyset$. This is possible because, for $x=r \xi^{0}+y+z$ with $|y| \leqslant r \delta$ and $|z|<1$, we have $r(1-\delta)-1 \leqslant|x| \leqslant r(1+\delta)+1$ and

$$
\left|\frac{x}{|x|}-\xi_{0}\right|=\frac{\left|(r-|x|) \xi^{0}+y+z\right|}{|x|} \leqslant \frac{2(r \delta+1)}{r(1-\delta)-1} \leqslant \frac{2\left(\delta+R^{-1}\right)}{1-\delta-R^{-1}},
$$

and $\xi^{0} \notin \overline{C_{\sigma}^{0}(n-1)}$.

(i) Assume that $\operatorname{dim} \operatorname{Span}\left\{\xi_{1}, \ldots, \xi_{l}\right\} \leqslant n-1$. We claim that, if $w=\sum_{j=1}^{l} r_{j} \xi_{j}$ with $r_{1}, \ldots, r_{l}>0$, then $w \in \Pi^{-1} \bigcup_{k=1}^{n-1} C_{\sigma}^{0}(k) \cup\{0\}$. Indeed, if $\xi_{1}, \ldots, \xi_{l}$ are linearly independent, then this is clear; if $\xi_{1}, \ldots, \xi_{l}$ are linearly dependent, then we can find (after change of numbering if necessary) $b_{1}, \ldots, b_{l}$ satisfying $\sum_{j=1}^{l} b_{j} r_{j} \xi_{j}=0$ and $b_{l}=1 \geqslant b_{j}$ for $1 \leqslant j \leqslant l-1$, which implies that $w$ is a linear combination with positive coefficients of some $\left\{\xi_{1}^{\prime}, \ldots, \xi_{l^{\prime}}^{\prime}\right\} \varsubsetneqq\left\{\xi_{1}, \ldots, \xi_{l}\right\}$. We can continue this procedure until $w$ is expressed as a linear combination with positive coefficients of some linearly independent subsystem of $\left\{\xi_{1}, \ldots, \xi_{l}\right\}$. Now we see that $I=0$ from the definition of $I$.

(ii) Let $|y| \leqslant \delta r / 2$ and $r \geqslant R$. Suppose that $w=\sum_{j=1}^{l} r_{j} \xi_{j} \in r \xi^{0}+y+B_{1}$. We may assume that there is $l_{1}$ such that $r_{j} \geqslant \delta r /(2 l)$ for $j=1, \ldots, l_{1}$ and $r_{j}<\delta r /(2 l)$ for $j=l_{1}+1, \ldots, l$ after rearrangement of $\xi_{1}, \ldots, \xi_{l}$ if necessary. Write $y_{2}=\sum_{j=l_{1}+1}^{l} r_{j} \xi_{j}, y_{1}=y-y_{2}$, and $w^{\prime}=w-y_{2}=\sum_{j=1}^{l_{1}} r_{j} \xi_{j}$. Then 
$\left|y_{2}\right| \leqslant \delta r / 2,\left|y_{1}\right| \leqslant|y|+\left|y_{2}\right| \leqslant \delta r$, and $w^{\prime} \in r \xi^{0}+y+B_{1}-y_{2}=r \xi^{0}+y_{1}+B_{1}$. Thus, $w^{\prime} \notin \Pi^{-1} \overline{C_{\sigma}^{0}(n-1)}$. Now the property shown in the proof of (i) tells us that $\operatorname{dim} \operatorname{Span}\left\{\xi_{1}, \ldots, \xi_{l_{1}}\right\} \geqslant n$. Hence $l_{1} \geqslant n$. It follows that $I$ is not larger than

$$
\left(\begin{array}{l}
l \\
n
\end{array}\right)\left(\frac{\delta r}{2 l}\right)^{-(1+\alpha) n} \int_{(\theta, \infty)^{l-n}}\left(\prod_{j=n+1}^{l} \frac{d r_{j}}{r_{j}^{1+\alpha}}\right) \int_{(\theta, \infty)^{n}} 1_{r \xi^{0}+y_{1}+B_{1}}\left(\sum_{j=1}^{l_{1}} r_{j} \xi_{j}\right) \prod_{j=1}^{n} d r_{j} .
$$

Denote by $V_{n}$ the volume of the $n$-dimensional unit ball. We have

$$
\int_{(\theta, \infty)^{n}} 1_{r \xi^{0}+y_{1}+B_{1}}\left(\sum_{j=1}^{l_{1}} r_{j} \xi_{j}\right) \prod_{j=1}^{n} d r_{j} \leqslant \frac{V_{n}}{D\left(\xi_{1}, \ldots, \xi_{n}\right)}
$$

when $r_{j}(n+1 \leqslant j \leqslant l)$ are fixed. Therefore, noting $V_{n} \leqslant 2^{n}$,

$$
J \leqslant\left(\begin{array}{l}
l \\
n
\end{array}\right)\left(\frac{\delta r}{2 l}\right)^{-(1+\alpha) n} \alpha^{-(l-n)} \theta^{-\alpha(l-n)} 2^{n} A_{n}
$$

which is written as (2.19). On the other hand, since $l_{1} \geqslant n$ it follows that $I$ is not larger than

$$
\left(\begin{array}{l}
l \\
n
\end{array}\right) \alpha^{-(n-1)}\left(\frac{\delta r}{2 l}\right)^{-\alpha(n-1)} \int_{(\theta, \infty)^{l-n}}\left(\prod_{j=n+1}^{l} \frac{d r_{j}}{r_{j}^{1+\alpha}}\right) \sup _{a \in \mathbb{R}^{d}} \int_{(\delta r) /(2 l)}^{\infty} 1_{a+B_{1}}\left(r_{1} \xi_{1}\right) \frac{d r_{1}}{r_{1}^{1+\alpha}} .
$$

We have by (1.6)

$$
\begin{aligned}
& \sup _{a \in \mathbb{R}^{d}} \int_{S^{d-1}} \sigma\left(d \xi_{1}\right) \int_{(\delta r) /(2 l)}^{\infty} 1_{a+B_{1}}\left(r_{1} \xi_{1}\right) \frac{d r_{1}}{r_{1}^{1+\alpha}} \\
= & r^{-\alpha} \sup _{a^{\prime} \in \mathbb{R}^{d}} \int_{S^{d-1}} \sigma\left(d \xi_{1}\right) \int_{\delta /(2 l)}^{\infty} 1_{a^{\prime}+B_{1 / r}}\left(u \xi_{1}\right) \frac{d u}{u^{1+\alpha}} \\
\leqslant & r^{-\alpha} \sup _{\xi \in S^{d-1}} \sigma\left(\xi+B_{\frac{2 l}{\delta r}}\right) \int_{\delta /(2 l)}^{\delta /(2 l)+2 / r} \frac{d u}{u^{1+\alpha}} \\
\leqslant & c_{3} \alpha^{-1}\left(\frac{2 l}{\delta}\right)^{\alpha} r^{-\alpha-1} \sigma^{*}\left(\frac{\delta r}{2 l}\right) \leqslant c_{4} l^{\alpha+b} r^{-\alpha-1} \sigma^{*}(r)
\end{aligned}
$$

with positive constants $b, c_{3}$ and $c_{4}$, which implies (2.20).

Remark 2.8. Let $K$ be a compact set in $T_{\sigma}(n)$. Then we see from the proof above the constants $c_{1}$ and $c_{2}$ can be taken uniformly with respect to $\xi^{0} \in K$.

\section{Proof of the main Results}

In this section, we prove the results mentioned in Section 1 and their corollaries and remarks by using the preliminaries in Section 2. Then we present some additional results which give the proper asymptotic orders of the stable densities. We continue to use the notation of Sections 1 and 2.

Lemma 3.1. (i) There is a constant $c_{1}>0$ such that

$$
\lambda\left(x+B_{1}\right) \leqslant c_{1}(1+|x|)^{-(1+\alpha)} \sigma^{*}(1+|x|) \quad \text { for } x \in \mathbb{R}^{d} .
$$

(ii) Let $\xi^{0} \in S^{d-1}$. There are constants $c_{2}>0$ and $R>0$ independent of $\xi^{0} \in S^{d-1}$ such that

$$
\lambda\left(r \xi^{0}+B_{2}\right) \geqslant c_{2}(1+r)^{-(1+\alpha)} \sigma_{\xi^{0}}(1+r) \quad \text { for } r \geqslant R .
$$


Proof. (i) Recall (1.4) and see that $\nu_{Z}(d x)=1_{\{|x|>\theta\}}(x) \nu(d x)$. Then, for $n \geqslant 1$ and $|x| \geqslant 2$,

$$
\nu_{Z}^{n *}\left(x+B_{1}\right)=c^{n} \int_{\left(S^{d-1}\right)^{n} \times(\theta, \infty)^{n}} 1_{x+B_{1}}\left(\sum_{j=1}^{n} r_{j} \xi_{j}\right) \prod_{k=1}^{n} r_{k}^{-(1+\alpha)} \sigma\left(d \xi_{k}\right) d r_{k} .
$$

If $\sum_{j=1}^{n} r_{j} \xi_{j} \in x+B_{1}$, then $r_{l} \geqslant|x| /(2 n)$ for some $l$ with $1 \leqslant l \leqslant n$. Thus we see from (1.6) that

$$
\begin{aligned}
& \nu_{Z}^{n *}\left(x+B_{1}\right) \leqslant c^{n} \alpha^{-(n-1)} \theta^{-\alpha(n-1)} \sup _{a \in \mathbb{R}^{d}} \int_{S^{d-1}} \sigma\left(d \xi_{l}\right) \int_{|x| /(2 n)}^{\infty} 1_{a+B_{1}}\left(r_{l} \xi_{l}\right) \frac{d r_{l}}{r_{l}^{1+\alpha}} \\
= & c^{n} \alpha^{-(n-1)} \theta^{-\alpha(n-1)}|x|^{-\alpha} \sup _{a^{\prime} \in \mathbb{R}^{d}} \int_{S^{d-1}} \sigma\left(d \xi_{l}\right) \int_{1 /(2 n)}^{\infty} 1_{a^{\prime}+B_{1 /|x|}}\left(u \xi_{l}\right) \frac{d u}{u^{1+\alpha}} \\
\leqslant & c^{n} \alpha^{-(n-1)} \theta^{-\alpha(n-1)}|x|^{-\alpha} \sup _{\xi \in S^{d-1}} \sigma\left(\xi+B_{\frac{2 n}{|x|}}\right) \int_{1 /(2 n)}^{1 /(2 n)+2 /|x|} \frac{d u}{u^{1+\alpha}} \\
\leqslant & c_{3} c^{n} \alpha^{-n} \theta^{-\alpha(n-1)}(2 n)^{\alpha}|x|^{-\alpha-1} \sigma^{*}\left(\frac{|x|}{2 n}\right) \\
\leqslant & c_{4} c^{n} \alpha^{-n} \theta^{-\alpha(n-1)}(2 n)^{\alpha+b}(1+|x|)^{-\alpha-1} \sigma^{*}(1+|x|)
\end{aligned}
$$

with some positive constants $c_{3}$ and $c_{4}$. Hence there is $c_{5}$ such that

$\lambda\left(x+B_{1}\right) \leqslant \sum_{n=1}^{\infty} e^{-a}(n !)^{-1} \nu_{Z}^{n *}\left(x+B_{1}\right) \leqslant c_{5}(1+|x|)^{-(1+\alpha)} \sigma^{*}(1+|x|) \quad$ for $|x| \geqslant 2$,

where $a=\nu_{Z}\left(\mathbb{R}^{d}\right)$. This proves (3.1) with some $c_{1}$.

(ii) Let $r>2 \theta+2$. There is $c_{6}>0$ independent of $\xi^{0} \in S^{d-1}$ such that

$$
\begin{aligned}
& \lambda\left(r \xi^{0}+B_{2}\right) \geqslant e^{-a} \nu_{Z}\left(r \xi^{0}+B_{2}\right) \\
= & e^{-a} \int_{S^{d-1}} \sigma\left(d \xi_{1}\right) \int_{\theta}^{\infty} 1_{r \xi^{0}+B_{2}}\left(r_{1} \xi_{1}\right) \frac{d r_{1}}{r_{1}^{1+\alpha}} \\
= & e^{-a} r^{-\alpha} \int_{S^{d-1}} \sigma\left(d \xi_{1}\right) \int_{\theta / r}^{\infty} 1_{\xi^{0}+B_{2 / r}}\left(u \xi_{1}\right) \frac{d u}{u^{1+\alpha}} \\
\geqslant & c_{6} e^{-a} \alpha^{-1} r^{-\alpha-1} \sigma\left(\xi^{0}+B_{1 / r}\right) \\
\geqslant & c_{6} e^{-a} \alpha^{-1}(1+r)^{-\alpha-1} \sigma_{\xi^{0}}(1+r),
\end{aligned}
$$

which implies (3.2).

Proof of Theorem 1.1. (i) By Lemma 3.1 and (1.6) we can find $c_{5}>0$ and $c_{6}>0$ such that, for any $x, z \in \mathbb{R}^{d}$ with $|z| \leqslant|x| / 2$,

$\lambda\left(x+z+B_{1}\right) \leqslant c_{5}(1+|x+z|)^{-(1+\alpha)} \sigma^{*}(1+|x+z|) \leqslant c_{6}(1+|x|)^{-(1+\alpha)} \sigma^{*}(1+|x|)$.

Hence, applying Lemma 2.5 (i) to $K_{1}=S^{d-1}$ and $y=0$, we get (1.9). The second assertion is obviously true because $\sigma^{*}(1+|x|) \leqslant 1$ for $x \in \mathbb{R}^{d}$.

(ii) By Lemma 3.1 we can find $c_{7}>0$ and $R_{3}>0$ such that

$$
\lambda\left(r \xi^{0}+B_{2}\right) \geqslant c_{7}(1+r)^{-\alpha-1} \sigma_{\xi^{0}}(1+r) \text { for } r>R_{3} .
$$

Hence, applying Lemma 2.5 (ii) to $K_{2}=\left\{\xi^{0}\right\}$ we get (1.11). 
(iii) We have $K_{1} \subset C_{\sigma}^{0}$. Let us check the assumption in Lemma 2.5 (ii) with $\varphi_{2}(r)=r^{-d(1+\alpha)}$. Namely, we claim that there are $c_{8}>0$ and $R_{4}>0$ such that if $\xi \in K_{1}$ and $r \geqslant R_{4}$, then

$$
\lambda\left(r \xi+B_{1}\right) \geqslant c_{8}(1+r)^{-d(1+\alpha)} .
$$

Let $\xi^{0} \in K_{1}$. By (1.7) we can find a linearly independent system $\xi_{1}^{0}, \ldots, \xi_{d}^{0}$ in $S_{\sigma}$ and positive reals $a_{1}^{0}, \ldots, a_{d}^{0}$ such that $\xi^{0}=\sum_{j=1}^{d} a_{j}^{0} \xi_{j}^{0}$. Choose a small relatively open neighborhood $U\left(\xi_{j}^{0}\right)$ of $\xi_{j}^{0}$ in $S^{d-1}$ for each $j$ in such a way that, for every $\xi_{j} \in U\left(\xi_{j}^{0}\right), j=1, \ldots, d$, there uniquely exist $a_{j}>0, j=1, \ldots, d$, satisfying $\xi^{0}=\sum_{j=1}^{d} a_{j} \xi_{j}$ and that $a_{1}, \ldots, a_{d}$ continuously depend on $\left\{\xi_{j}\right\}$. We have $a_{j}=a_{j}^{0}$ for $\left\{\xi_{j}\right\}=\left\{\xi_{j}^{0}\right\}$. For $r>2$ let

$V_{\varepsilon}\left(r \xi^{0}\right)=\left\{y=\sum_{j=1}^{d} s_{j} \xi_{j}: \xi_{j} \in U\left(\xi_{j}^{0}\right)\right.$ and $a_{j} r-\varepsilon<s_{j}<a_{j} r+\varepsilon$ for $\left.j=1, \ldots, d\right\}$.

Choose $\varepsilon>0$ so small that $V_{\varepsilon}\left(r \xi^{0}\right) \subset r \xi^{0}+B_{1}$. Then, with $a=\nu_{Z}\left(\mathbb{R}^{d}\right)$, there are $c_{9}$ and $R_{5}$ such that

$$
\begin{aligned}
\lambda\left(r \xi^{0}+B_{1}\right) & \geqslant e^{-a}(d !)^{-1} \nu_{Z}^{d *}\left(V_{\varepsilon}\left(r \xi^{0}\right)\right) \\
& \geqslant e^{-a}(d !)^{-1} c^{d} \int_{U\left(\xi_{1}^{0}\right) \times \cdots \times U\left(\xi_{d}^{0}\right)} \sigma\left(d \xi_{1}\right) \cdots \sigma\left(d \xi_{d}\right) \prod_{j=1}^{d} \int_{a_{j} r-\varepsilon}^{a_{j} r+\varepsilon} s_{j}^{-(1+\alpha)} d s_{j} \\
& \geqslant c_{9} r^{-d(1+\alpha)} \geqslant c_{9}(1+r)^{-d(1+\alpha)}
\end{aligned}
$$

for all $r \geqslant R_{5}$. The set $C_{\sigma}^{0}$ is relatively open in $S^{d-1}$. On reflection we see that there is a small relatively open neighborhood $W\left(\xi^{0}\right)$ of $\xi^{0}$ in $S^{d-1}$ such that we can find $c_{10}$ and $R_{6}$ satisfying

$$
\lambda\left(r \xi+B_{1}\right) \geqslant c_{10}(1+r)^{-d(1+\alpha)}
$$

for all $\xi \in W\left(\xi^{0}\right)$ and $r \geqslant R_{6}$. By compactness of $K_{1}$ we see (3.3). Now we have only to apply Lemma 2.5 (ii).

(iv) This is a one-sided case. Given $K_{2}, c_{0}$, and $\delta_{3}$ with $K_{2} \cap C_{\sigma}=\emptyset$, we have to show (1.13). Let $\delta$ be the distance between $K_{2}$ and $C_{\sigma}$. We have $0<\delta<2$. Choosing $0<\theta<\delta /\left(8 c_{0}\right)$, we will apply Lemma 2.4. Let $x /|x| \in K_{2}$ and $|y| \leqslant \delta_{2}$. As in the proof of Lemma 2.5 (i),

$$
p(x+y)=\int_{|z| \leqslant \delta|x| / 4} q(-z) \lambda(x+y+d z)+\int_{|z|>\delta|x| / 4} q(-z) \lambda(x+y+d z) .
$$

Denote by $J_{1}$ and $J_{2}$ the first and the second integral. As before, we have $J_{1} \leqslant$ $\sum_{j=1}^{N} Q_{j} \lambda\left(x+y+x_{j}+B_{1}\right)$ where $\left|x_{j}\right| \leqslant \delta|x| / 4$. But $\left(x+y+x_{j}+B_{1}\right) \cap \Pi^{-1} C_{\sigma}=\emptyset$ uniformly in $y$ and $x_{j}$ when $|x|$ is large. Indeed, letting $\left|y-y^{\prime}\right|<1$,

$$
\begin{aligned}
& \left|\Pi\left(x+y^{\prime}+x_{j}\right)-\Pi x\right| \leqslant|| x+y^{\prime}+\left.x_{j}\right|^{-1}-|x|^{-1}|| x|+| x+y^{\prime}+\left.x_{j}\right|^{-1}\left|y^{\prime}+x_{j}\right| \\
& \quad \leqslant 2\left|x+y^{\prime}+x_{j}\right|^{-1}\left|y^{\prime}+x_{j}\right| \\
& \quad \leqslant\left(|x|-\delta|x| / 4-\left|y^{\prime}\right|\right)^{-1}\left(\delta|x| / 2+2\left|y^{\prime}\right|\right) \rightarrow(1-\delta / 4)^{-1} \delta / 2<\delta
\end{aligned}
$$

as $|x| \rightarrow \infty$. It follows from (2.14) that $J_{1}=0$ when $|x|$ is large. Now we get (1.13) for large $|x|$ from Lemma 2.4. If we take the constant $c_{4}$ large enough, it holds without restriction that $|x|$ is large. 
Remark 3.2. A nondegenerate $\alpha$-semistable process with $0<\alpha<2$ on $\mathbb{R}^{d}$ does not satisfy (1.10) in general. We give such an example for $d=1$. However, it is noteworthy that the description of the finiteness of the moment of the last exit time from a ball in Corollary 2.7 of Sato and Watanabe 34 in one-dimensional case is true for all semistable processes as well as stable processes.

Proof. Let $d=1$ and $0<\alpha<2$. We give an example of an $\alpha$-semistable process on $\mathbb{R}$ which does not satisfy (1.10). Let $a>1$ and $\nu=\sum_{n=-\infty}^{\infty} a^{-n} \delta_{a^{n / \alpha}}(d x)$ be the process' Lévy measure, where $\delta_{a^{n / \alpha}}$ is the $\delta$-measure at $a^{n / \alpha}$. That is, $\nu$ is a discrete one-sided measure. In the case of $0<\alpha<1$, we assume that the drift of the process is nonpositive. Then the associated $\alpha$-semistable process does not satisfy (1.10). Indeed, choose $\theta=1$ in the same decomposition $\left\{X_{t}\right\} \stackrel{\mathrm{d}}{=}\left\{Y_{t}+Z_{t}\right\}$ as for the stable case. Then $c=\nu_{Z}(\mathbb{R})=1 /(a-1)$ and, letting $0<\varepsilon<1$ and $n \geqslant 1$, we have $\lambda\left(\left(a^{n / \alpha}-\varepsilon, a^{n / \alpha}+\varepsilon / 2\right)\right) \geqslant e^{-c} c a^{-n}$. Note that the density $q(x)=q(1, x)$ of $\mathcal{L}\left(Y_{1}\right)$ is continous and positive on $[0, \infty)$. See Proposition 3.7 of 34 . Thus

$$
\int_{a^{n / \alpha}-\varepsilon}^{a^{n / \alpha}+\varepsilon} p(x) d x \geqslant \int_{0}^{\varepsilon / 2} q(y) d y \lambda\left(\left(a^{n / \alpha}-\varepsilon, a^{n / \alpha}+\varepsilon / 2\right)\right) \geqslant c_{2} a^{-n}
$$

with some $c_{2}>0$. Hence, if (1.10) holds, we have

$$
0<\liminf _{n \rightarrow \infty} a^{n} \int_{a^{n / \alpha}-\varepsilon}^{a^{n / \alpha}+\varepsilon} p(x) d x \leqslant \liminf _{n \rightarrow \infty} a^{n} c_{1}\left(a^{n / \alpha}-\varepsilon+1\right)^{-1-\alpha} 2 \varepsilon=0,
$$

which is absurd.

Remark 3.3. Let $d \geqslant 2$.

(i) Suppose that there are $\xi^{0} \in S_{\sigma}$ and $c_{1}>0$ such that

$$
\sigma^{*}(1+r) \leqslant c_{1} \sigma_{\xi^{0}}(1+r) \quad \text { for } r>0 .
$$

Then, given $\delta_{1}>0$ there are $c_{2}>0$ and $c_{3}>0$ such that

$$
c_{2}(1+r)^{-(1+\alpha)} \sigma_{\xi^{0}}(1+r) \leqslant p\left(r \xi^{0}+y\right) \leqslant c_{3}(1+r)^{-(1+\alpha)} \sigma_{\xi^{0}}(1+r)
$$

whenever $|y| \leqslant \delta_{1}$.

(ii) Fix $s \in(0, d-1)$. Let $\ell(r)$ be slowly varying as $r \rightarrow \infty$ and define $\ell^{*}(r)=$ $\int_{r}^{\infty} \ell(u) d u / u$ in case $\int_{1}^{\infty} \ell(u) d u / u<\infty$. Then we can choose $\sigma$ such that it is absolutely continuous with respect to $m, \sigma_{\xi^{0}}(r)$ satisfies (3.4), and $\sigma_{\xi^{0}}(r) \asymp r^{-s} \ell(r)$ or $\sigma_{\xi^{0}}(r) \asymp \ell^{*}(r)$. In general, $A(t) \asymp B(t)$ means that there are constants $0<c_{3} \leqslant$ $c_{4}<\infty$ such that $c_{3} B(t) \leqslant A(t) \leqslant c_{4} B(t)$ for all sufficiently large $t$.

Proof. Assertion (i) is obvious from (i) and (ii) of Theorem 1.1. Next we prove Assertion (ii). Fix $s \in(0, d-1)$ and let $\ell(r)$ be slowly varying as $r \rightarrow \infty$. Let $h(u)$ be a nonnegative measurable function on $(0,2]$ such that $h(0+)=\infty$ and $h(u)$ is bounded on $(\delta, 2]$ for every $\delta \in(0,2)$. Then we can choose $\sigma$ by $\sigma(d \xi)=$ $h\left(\left|\xi-\xi^{0}\right|\right) m(d \xi)$ satisfying $h(1 / r) \asymp r^{d-1-s} \ell(r)$. Then we have $\sigma_{\xi^{0}}(r) \asymp r^{-s} \ell(r)$. In the same way, if $\int_{1}^{\infty} \ell(u) d u / u<\infty$, then we can set $\sigma$ by $\sigma(d \xi)=h\left(\left|\xi-\xi^{0}\right|\right) m(d \xi)$ with $h(1 / r) \asymp r^{d-1} \ell(r)$. Then we get $\sigma_{\xi^{0}}(r) \asymp \ell^{*}(r)$. Moreover, the measures $\sigma$ fulfill (3.4) because $h(0+)=\infty$ and $h(u)$ is bounded on $(\delta, 2]$ for every $\delta \in(0,2)$.

Next we observe more precisely both of the best possible and the worst possible cases as corollaries of Theorem 1.1. 
Corollary 3.4. Let $\xi^{0} \in S_{\sigma}$. In the case where $0<\alpha<1$ and $\nu$ is one-sided, we make an additional assumption that $\xi^{0} \in \operatorname{int} C_{\sigma}$. Suppose that $\sigma\left(\left\{\xi^{0}\right\}\right)>0$. Then, given $\delta>0$, we can find $c_{1}>0$ and $c_{2}>0$ such that

$$
c_{1}(1+r)^{-(1+\alpha)} \leqslant p\left(r \xi^{0}+y\right) \leqslant c_{2}(1+r)^{-(1+\alpha)} \quad \text { whenever }|y| \leqslant \delta .
$$

Proof. Since $\lim _{r \rightarrow \infty} \sigma_{\xi^{0}}(r)=\sigma\left(\left\{\xi^{0}\right\}\right)>0$, the corollary is clear from (1.10) and (1.11).

Corollary 3.5. Let $d \geqslant 2$. Consider a compact set $K \subset T_{\sigma}(d)$. Define the set $\Xi_{d}$ as $\Xi_{d}=\left\{\left(\xi_{1}, \ldots, \xi_{d}\right): \operatorname{det}\left(\xi_{1}, \ldots, \xi_{d}\right) \neq 0, \xi_{j} \in S^{d-1}, j=1, \ldots, d\right\}$ where $\operatorname{det}\left(\xi_{1}, \ldots, \xi_{d}\right)$ is the determinant considering each $\xi_{j}$ as a column vector in $\mathbb{R}^{d}$. Suppose that $\sigma=\sum_{j=1}^{N} \sigma_{j}$ with $N \geqslant d$ where $S_{\sigma_{j}}=S^{d-1} \cap C_{j}$ with $C_{j}$ being a nonempty closed convex cone in $\mathbb{R}^{d}$ and $S_{\sigma_{i}} \cap S_{\sigma_{j}}=\emptyset$ for $i \neq j$. Further suppose that, for any set $\{k(j)\}_{j=1}^{d}$ of strictly increasing integers with $1 \leqslant k(j) \leqslant N$,

$$
\int_{\Xi_{d}} \frac{1}{\left|\operatorname{det}\left(\xi_{1}, \ldots, \xi_{d}\right)\right|} \prod_{j=1}^{d} \sigma_{k(j)}\left(d \xi_{j}\right)<\infty .
$$

Then, given $\delta_{1}>0$, we can find $c_{1}>0$ and $c_{2}>0$ such that

$$
c_{1}(1+|x|)^{-(1+\alpha) d} \leqslant p(x+y) \leqslant c_{2}(1+|x|)^{-(1+\alpha) d}
$$

whenever $x /|x| \in K,|y| \leqslant \delta_{1}$.

Proof. The left-hand side inequality in (3.8) is true by (1.12) in Theorem 1.1 with $K_{1}=K$. Let $\delta$ and $R$ be those discovered in Lemma 2.7 Let $|y| \leqslant \delta r / 2$ and $r \geqslant R$. By Lemma 2.7 (i),

$$
\lambda\left(r \xi^{0}+y+B_{1}\right)=\sum_{l=d}^{\infty} e^{-a}(l !)^{-1} \nu_{Z}^{l *}\left(r \xi^{0}+y+B_{1}\right) .
$$

Let $n=d$ in the proof of Lemma 2.7 (ii). Then we can express $w^{\prime}$ by the convexity of $C_{j}, 1 \leqslant j \leqslant N$, as

$$
w^{\prime}=\sum_{j=1}^{l_{1}} r_{j} \xi_{j}=\sum_{j=1}^{l_{2}} s_{j} \zeta_{j}
$$

where $1 \leqslant l_{2} \leqslant l_{1}$ and $s_{j}>0$ for $1 \leqslant j \leqslant l_{2}$ and $\zeta_{j} \in S_{\sigma_{k(j)}}$ for strictly increasing integers $\{k(j)\}_{j=1}^{l_{2}}$ with $1 \leqslant k(j) \leqslant N$. Hence, by $w^{\prime} \notin \Pi^{-1}\left(\overline{C_{\sigma}^{0}(d-1)}\right)$, we have $l_{1} \geqslant l_{2} \geqslant d$ and we may assume that $\xi_{j} \in S_{\sigma_{k(j)}}$ for $1 \leqslant j \leqslant d$ with $\{k(j)\}_{j=1}^{d}$ as above. We have, for $l \geqslant d$,

$$
\begin{aligned}
\nu_{Z}^{l *}\left(r \xi^{0}+y+B_{1}\right) & =c^{l} J\left(l, r, \xi^{0}, y\right) \\
& \leqslant c^{l} c_{1}\left(\begin{array}{l}
l \\
d
\end{array}\right) l^{(1+\alpha) d} \alpha^{-l} \theta^{-\alpha l} r^{-(1+\alpha) d} A,
\end{aligned}
$$

where $c_{1}$ is a positive constant independent of $\xi^{0} \in K$ and $A$ is the maximum of the integral in (3.7) in all possible $\{k(j)\}_{j=1}^{d}$. Here $c$ is the constant in (1.4). Summing up in $l$, we get

$$
\sup _{\xi^{0} \in K} \lambda\left(r \xi^{0}+y+B_{1}\right) \leqslant c_{3} r^{-(1+\alpha) d} \leqslant c_{2}(1+r)^{-(1+\alpha) d}
$$

with some $c_{3}$ and $c_{2}$. Hence, applying Lemma 2.5 (i) for $K_{1}=K$ and $\delta_{0}=\delta / 2$, we get the right-hand side inequality in (3.8). 
Proof of Theorem 1.2. The right-hand side inequality is true by (i) of Theorem 1.1 and (1.14). We show the left-hand side. Let $\xi^{0}=\xi \in K$ in Lemma 3.1. Then we have

$$
\liminf _{r \rightarrow \infty} \inf _{\xi^{0} \in K} \frac{\lambda\left(r \xi^{0}+B_{2}\right)}{(1+r)^{-(1+\alpha)} \phi(1+r)} \geqslant c_{3} \liminf _{r \rightarrow \infty} \inf _{\xi^{0} \in K} \frac{\sigma_{\xi^{0}}(1+r)}{\phi(1+r)}
$$

with some $c_{3}>0$. It follows from (1.14) that there are $c_{4}>0$ and $R_{1}>0$ such that

$$
\lambda\left(r \xi^{0}+B_{2}\right) \geqslant c_{4}(1+r)^{-(1+\alpha)} \phi(1+r)
$$

for $\xi^{0} \in K$ and $r \geqslant R_{1}$. Thus, letting $K_{2}=K$ and $\delta_{2}=\delta$, we obtain from Lemma 2.5 (ii) that there are $c_{1}>0$ and $c_{2}>0$ such that the left-hand side inequality holds.

Remark 3.6. Let $E$ be the classical Cantor set with Hausdorff dimension $\log 2 / \log 3$ in $[0,1]$. We identify $S^{1}$ with $[0,2 \pi)$ by the usual map $\xi=\xi(\theta), \theta \in[0,2 \pi)$. Let $d=2$ and set $K=\xi(E)$ and $s=\log 2 / \log 3$ in Remark 1.3. Then we have $C_{\sigma}=\xi([0,1])$ and $\beta=1+\alpha+\log 2 / \log 3$ and the following.

(i) In the case where $0<\alpha<1$, we make an additional assumption that $0<$ $\theta<1$. If $\theta \in E$, then there are some positive constants $c_{1}, c_{2}$, and $\delta_{1}$ such that

$$
c_{1}(1+r)^{-\beta} \leqslant p(r \xi(\theta)+y) \leqslant c_{2}(1+r)^{-\beta} \text { whenever }|y| \leqslant \delta_{1} .
$$

(ii) If $\theta \notin E$ and $0<\theta<1$, then there are some positive constants $c_{3}, c_{4}$, and $\delta_{2}$ such that

$$
c_{3}(1+r)^{-2-2 \alpha} \leqslant p(r \xi(\theta)+y) \leqslant c_{4}(1+r)^{-2-2 \alpha} \quad \text { whenever }|y| \leqslant \delta_{2} .
$$

the proof of assertion (ii) is similar to that of Corollary 3.5. In this example, the surface $\left\{(x, y): y=p(x), x \in \mathbb{R}^{2}\right\}$ in $\mathbb{R}^{3}$ is waving infinitely many times when $|x|$ is sufficiently large. Thus it is a delicately difficult problem whether all multivariate stable distributions are unimodal in a certain sense as a natural extension of the univariate unimodality proved by Yamazato [42. See also Kanter [17, Watanabe [40] and Wolfe [4] for the symmetric case.

Proof of Theorem 1.4. (i) Let $\delta$ and $R$ be those discovered in Lemma 2.7. Let $|y| \leqslant \delta r / 2$ and $r \geqslant R$. By Lemma 2.7 (i),

$$
\lambda\left(r \xi^{0}+y+B_{1}\right)=\sum_{l=n}^{\infty} e^{-a}(l !)^{-1} \nu_{Z}^{l *}\left(r \xi^{0}+y+B_{1}\right) .
$$

We have as in the proof of $(3.1)$, for $l \geqslant n$,

$$
\begin{aligned}
\nu_{Z}^{l *}\left(r \xi^{0}+y+B_{1}\right) & =c^{l} J\left(l, r, \xi^{0}, y\right) \\
& \leqslant c^{l} c_{3}\left(\begin{array}{l}
l \\
n
\end{array}\right) l^{\alpha n+b} \alpha^{-l} \theta^{-\alpha l} r^{-(1+\alpha n)} \sigma^{*}(r) \\
& \leqslant c^{l} c_{4}\left(\begin{array}{l}
l \\
n
\end{array}\right) l^{\alpha n+b} \alpha^{-l} \theta^{-\alpha l}(1+r)^{-(1+\alpha n)} \phi_{1}(1+r)
\end{aligned}
$$

with some $c_{3}$ and $c_{4}$ by Lemma 2.7 (ii). Here $c$ is the constant in (1.4). Summing up in $l$, we get

$$
\lambda\left(r \xi^{0}+y+B_{1}\right) \leqslant c_{5}(1+r)^{-(1+\alpha n)} \phi_{1}(1+r)
$$

with some $c_{5}$. Thus by Lemma 2.5 (i) we have (1.19). 
(ii) We express $\xi^{0}$ as $\xi^{0}=\sum_{j=1}^{n} a_{j} \xi_{j}^{0}$ with $a_{j}>0$ and $\xi_{j}^{0} \in S_{\sigma}$ for $1 \leqslant j \leqslant n$. Then $\sum_{j=1}^{n} r_{j} \xi_{j}-r \xi^{0} \in B_{1}$ if $\left|r_{j}-r a_{j}\right|<1 /(3 n)$ and $\left|\xi_{j}-\xi_{j}^{0}\right|<\left(2\left(a_{j}+1\right) n(1+r)\right)^{-1}$. Hence

$$
\begin{aligned}
\lambda\left(r \xi^{0}+B_{1}\right) & \geqslant e^{-a}(n !)^{-1} \nu_{Z}^{n *}\left(r \xi^{0}+B_{1}\right) \\
& \geqslant e^{-a}(n !)^{-1} c^{n}\left(\prod_{j=1}^{n} \sigma\left(\xi_{j}^{0}+B \frac{1}{2\left(a_{j}+1\right) n(1+r)}\right)\right) \prod_{j=1}^{n} \int_{r a_{j}-1 /(3 n)}^{r a_{j}+1 /(3 n)} r_{j}^{-(1+\alpha)} d r_{j} \\
& \geqslant c_{6}\left((1+r)^{-(1+\alpha)} \phi_{2}(1+r)\right)^{n}
\end{aligned}
$$

with some positive $c_{6}$ for $r \geqslant a_{j}^{-1}(\theta+1 / n), 1 \leqslant j \leqslant n$. It follows from Lemma 2.5 (ii) that, for some $c_{4}>0,(1.21)$ holds.

Proof of Theorem 1.5. (i) Suppose that (1.22) holds. Then there are $c_{3}>0$ and $c_{4}>0$ such that

$$
\sigma^{*}(r)=\sup _{\xi \in S^{d-1}} \sigma\left(\xi+B_{1 / r}\right) \leqslant c_{3} m\left(\xi+B_{1 / r}\right) \leqslant c_{4} r^{-(d-1)} .
$$

Thus we have by Theorem 1.1 (i)

$$
p(x) \leqslant c_{2}(1+|x|)^{-(1+\alpha)}(1+|x|)^{-(d-1)}=c_{2}(1+|x|)^{-(d+\alpha)} .
$$

Let us prove the converse, excepting the case where $0<\alpha<1$ and $\nu$ is one-sided. Suppose that (1.23) holds. Then by Theorem 1.1 (ii) we see that, for any $\xi \in S^{d-1}$,

$$
\liminf _{r \rightarrow \infty} \frac{\sigma\left(\xi+B_{1 / r}\right)}{r^{-(d-1)}} \leqslant c_{5} \liminf _{r \rightarrow \infty} p(r \xi) r^{d+\alpha} \leqslant c_{6}
$$

with some positive constants $c_{5}$ and $c_{6}$ independent of $\xi \in S^{d-1}$. Thus we obtain from Theorem 2.12 of Mattila 21] that $\sigma$ is absolutely continuous with respect to $m$ and the derivative $d \sigma / d m$ is bounded.

(ii) Let $\xi \in K$. For all large $r$, we have

$$
\begin{aligned}
\nu_{Z}(r \xi & \left.+B_{1}\right)=\nu\left(r \xi+B_{1}\right)=r^{-\alpha} \nu\left(\xi+B_{1 / r}\right) \\
& =r^{-\alpha} c \int_{S^{d-1}} \sigma\left(d \xi^{\prime}\right) \int_{0}^{\infty} 1_{\xi+B_{1 / r}}\left(u \xi^{\prime}\right) u^{-1-\alpha} d u \\
& \geqslant r^{-\alpha-1} c_{7} \sigma\left(\xi+B_{1 /(2 r)}\right) \geqslant c_{8} r^{-(d+\alpha)}
\end{aligned}
$$

with some $c_{7}>0$ and $c_{8}>0$, using (1.24). These constants do not depend on $\xi$ in $K$. Hence there are $c_{9}>0$ and $R>0$ such that

$$
\lambda\left(r \xi+B_{1}\right) \geqslant e^{-a} \nu_{Z}\left(r \xi+B_{1}\right) \geqslant c_{9}(1+r)^{-(d+\alpha)} \quad \text { for } \xi \in K \text { and } r \geqslant R .
$$

Therefore we get (1.25) from Lemma 2.5(ii).

The following remark is of some interest.

Remark 3.7. Let $d \geqslant 2$ and $\delta>0$.

(i) If the measure $\sigma$ is continuous, that is, if $\sigma(\{\xi\})=0$ for any $\xi \in S^{d-1}$, then

$$
\liminf _{r \rightarrow \infty} r^{1+\alpha} p(r \xi+y)=0 \quad \text { for each } \xi \in S^{d-1} \text { and every } y \text { with }|y| \leqslant \delta .
$$

The converse is also true except in the case where $0<\alpha<1$ and $\nu$ is one-sided.

(ii) Suppose that

$$
\liminf _{r \rightarrow \infty} r^{d+\alpha} p(r \xi+y)=0 \quad \text { for each } \xi \in S^{d-1} \text { and every } y \text { with }|y| \leqslant \delta .
$$


Then we have $0<\alpha<1, \nu$ is one-sided and $S_{\sigma} \subset \partial C_{\sigma}$ where $\partial C_{\sigma}$ is the boundary of the set $C_{\sigma}$ in the relative topology on $S^{d-1}$, and thereby $\sigma$ is singular with respect to $m$.

Proof. Assertion (i) is proved as follows. Assume that $\sigma$ is continuous and

$$
\lim _{r \rightarrow \infty} \sigma^{*}(r)>0 \text {. }
$$

Then there are $c>0, \xi_{n} \in S^{d-1}$ and $r_{n}>0$ such that $\xi_{n} \rightarrow \xi \in S^{d-1}$ and $r_{n} \uparrow \infty$ as $n \rightarrow \infty$ and $\sigma_{\xi_{n}}\left(r_{n}\right) \geqslant c$ for any $n \geqslant 1$. Then we have a contradiction :

$$
c \leqslant \lim _{k \rightarrow \infty} \limsup _{n \rightarrow \infty} \sigma_{\xi_{n}}\left(r_{k}\right) \leqslant \lim _{k \rightarrow \infty} \sigma_{\xi}\left(r_{k} / 2\right)=\sigma(\{\xi\})=0 .
$$

Thus, if $\sigma$ is continuous, then $\liminf _{r \rightarrow \infty} \sigma^{*}(r)=0$ and thereby (3.11) holds from (1.9). If $\liminf _{r \rightarrow \infty} r^{1+\alpha} p(r \xi+y)=0$ for each $\xi \in S^{d-1}$ and every $y$ with $|y| \leqslant \delta$ except in the case where $0<\alpha<1$ and $\nu$ is one-sided, then $\sigma(\{\xi\})=\liminf _{r \rightarrow \infty} \sigma_{\xi}(r)=0$ for each $\xi \in S^{d-1}$ by (1.11).

(ii) Suppose that (3.12) holds for $d \geqslant 2$. Let $\phi_{2}(r)=r^{-(d-1)}$. We have, by using Theorem 2.12 of Mattila [21] as in the proof of (i) of Theorem 1.5, that $\sigma=0$ except in the case where $0<\alpha<1$ and $\nu$ is one-sided. Next suppose that $0<\alpha<1$ and $\nu$ is one-sided. Then by Theorem 1.5 (ii) with $K=\{\xi\} \subset \operatorname{int} C_{\sigma}$ we see that

$$
\liminf _{r \rightarrow \infty} \frac{\sigma\left(\xi+B_{1 / r}\right)}{\phi_{2}(r)}=0 \quad \text { for any } \xi \in \operatorname{int} C_{\sigma} \text {. }
$$

Thus we again find from Theorem 2.12 of Mattila 21] that $\sigma=0$ on int $C_{\sigma}$. The last assertion is clear from $m\left(\partial C_{\sigma}\right)=0$.

Finally we can give the proper asymptotic order of $p(x+y)$ as $|x| \rightarrow \infty$ when $\sigma$ is absolutely continuous with respect to $m$, or when $S_{\sigma}$ is a finite set, or when $\sigma$ is the sum of such. Let $U$ be a relatively open set in $S^{d-1}$. Taking Carathéodory's theorem into account again, we define

$$
\begin{aligned}
& C^{0}(U, n)=\left\{\xi \in S^{d-1}: \xi=\sum_{j=1}^{n} c_{j} \xi_{j} \text { for some } c_{j}>0 \text { and } \xi_{j} \in U,\right. \\
&\left.j=1, \ldots, n, \text { such that } \xi_{1}, \ldots, \xi_{n} \text { are linearly independent }\right\}
\end{aligned}
$$

for $1 \leqslant n \leqslant d, T(U, 1)=C^{0}(U, 1)=U$, and

$$
T(U, n)=C^{0}(U, n) \backslash \overline{C^{0}(U, n-1)}
$$

for $2 \leqslant n \leqslant d$. If $S_{\sigma}=\bar{U}$, then $\overline{C^{0}(U, n)}=\overline{C_{\sigma}^{0}(n)}$ and hence $T(U, n) \subset T_{\sigma}(n)$ for $1 \leqslant n \leqslant d$; further in general $\bigcup_{n=1}^{d} T(U, n) \subset$ int $C_{\sigma}$.

Theorem 3.8. Let $U$ be a relatively open set in $S^{d-1}$ and let $K$ be a compact subset of $U$. Suppose that, for some $c_{1}>0$,

$$
\sigma(d \xi) \leqslant c_{1} m(d \xi) \quad \text { on } U, \quad \text { and } \sigma\left(U^{c}\right)=0
$$

and

$$
\liminf _{r \rightarrow \infty} \inf _{\xi \in K} \frac{\sigma\left(\xi+B_{1 / r}\right)}{r^{-(d-1)}}>0 \quad \text { for any } K \subset U \text {. }
$$

Assume that $\xi^{0} \in T(U, n)$ with $1 \leqslant n \leqslant d$. Then, given $\delta>0$, we can find $c_{2}>0$ and $c_{3}>0$ such that

$$
c_{2}(1+r)^{-(d+\alpha n)} \leqslant p\left(r \xi^{0}+y\right) \leqslant c_{3}(1+r)^{-(d+\alpha n)} \quad \text { whenever }|y| \leqslant \delta .
$$


Proof. For $n=1$, the theorem is true by Theorem 1.5. Suppose that $2 \leqslant n \leqslant d$, all assumptions of the theorem hold and that $\xi^{0} \in T(U, n)$ with $2 \leqslant n \leqslant d$. Then, by Theorem 1.4 (i), we have the right-hand side inequality with $\phi_{1}(r)=r^{-(d-1)}$. We can find a linearly independent system $\xi_{1}^{0}, \ldots, \xi_{n}^{0}$ in $U$ and positive reals $a_{1}^{0}, \ldots, a_{n}^{0}$ such that $\xi^{0}=\sum_{j=1}^{n} a_{j}^{0} \xi_{j}^{0}$. Choose big $R_{1}>0$, small $\varepsilon>0$, and a small relatively open neighborhood $U\left(\xi_{j}^{0}\right)$ of $\xi_{j}^{0}$ in $U$ for each $j$ in such a way that with $y=$ $r \xi^{0}-\sum_{j=2}^{n} x_{j}$

$$
\nu\left(d x_{j}\right) \geqslant c_{4} \frac{d x_{j}}{\left|x_{j}\right|^{d+\alpha}} \quad \text { on } U\left(\xi_{j}^{0}\right) \times\left(r\left(a_{j}^{0}-\varepsilon\right), r\left(a_{j}^{0}+\varepsilon\right)\right),
$$

for $2 \leqslant j \leqslant n$ and

$$
\nu\left(d x_{1}\right) \geqslant c_{5} \frac{d x_{1}}{\left|x_{1}\right|^{d+\alpha}} \quad \text { on } y+B_{1}
$$

with some positive $c_{4}$ and $c_{5}$. Further

$$
r a_{1}^{0} / 2 \leqslant|y| \leqslant 2 r a_{1}^{0} \quad \text { and } y+B_{1} \subset U \times(\theta, \infty)
$$

for $r \geqslant R_{1}$ and $x_{j} \in U\left(\xi_{j}^{0}\right) \times\left(r\left(a_{j}^{0}-\varepsilon\right), r\left(a_{j}^{0}+\varepsilon\right)\right)$ with $2 \leqslant j \leqslant n$. This is possible thanks to (3.16) and Theorem 2.12 of Mattila 21]. Let

$$
V_{j}=U\left(\xi_{j}^{0}\right) \times\left(r\left(a_{j}^{0}-\varepsilon\right), r\left(a_{j}^{0}+\varepsilon\right)\right)
$$

for $2 \leqslant j \leqslant n$. Then, with $a=\nu_{Z}\left(\mathbb{R}^{d}\right)$, there are $c_{6}, c_{7}$ and $R_{2}$ such that

$$
\begin{aligned}
\lambda\left(r \xi^{0}+B_{1}\right) & \geqslant e^{-a}(n !)^{-1} \nu_{Z}^{n *}\left(r \xi^{0}+B_{1}\right) \\
& \geqslant e^{-a}(n !)^{-1} c_{6}^{n} \int_{y+B_{1}}\left|x_{1}\right|^{-(d+\alpha)} d x_{1} \int_{V_{2} \times \cdots \times V_{n}} \prod_{j=2}^{n}\left|x_{j}\right|^{-(d+\alpha)} d x_{j} \\
& \geqslant c_{7} r^{-d-\alpha} r^{-\alpha(n-1)} \geqslant c_{7}(1+r)^{-(d+n \alpha)}
\end{aligned}
$$

for all $r \geqslant R_{2}$. Thus we obtain from Lemma 2.5 (ii) the left-hand side inequality of the theorem with the notice that $\bigcup_{n=1}^{d} T(U, n) \subset \operatorname{int} C_{\sigma}$.

Lemma 3.9. Assume that $S_{\sigma}$ is a finite set. Let $\xi^{0} \in T_{\sigma}(n)$ with $1 \leqslant n \leqslant d$. Then there are $c_{1}>0, c_{2}>0,0<\delta<1$ and $R>0$ such that

$$
\begin{aligned}
\lambda\left(r \xi^{0}+y+B_{1}\right) \leqslant c_{1}(1+r)^{-(1+\alpha) n} & \text { whenever }|y| \leqslant \delta r / 2 \text { and } r \geqslant R, \\
\lambda\left(r \xi^{0}+B_{1}\right) \geqslant c_{2}(1+r)^{-(1+\alpha) n} & \text { whenever } r \geqslant R .
\end{aligned}
$$

Proof. Let $\delta$ and $R$ be those discovered in Lemma 2.7 Let $|y| \leqslant \delta r / 2$ and $r \geqslant R$. By Lemma 2.7 (i),

$$
\lambda\left(r \xi^{0}+y+B_{1}\right)=\sum_{l=n}^{\infty} e^{-a}(l !)^{-1} \nu_{Z}^{l *}\left(r \xi^{0}+y+B_{1}\right) .
$$

We have

$$
\begin{aligned}
\nu_{Z}^{l *}\left(r \xi^{0}+y+B_{1}\right) & =c^{l} J\left(l, r, \xi^{0}, y\right) \\
& \leqslant c^{l} c_{3}\left(\begin{array}{l}
l \\
n
\end{array}\right) l^{(1+\alpha) n} \alpha^{-l} \theta^{-\alpha l} r^{-(1+\alpha) n} A_{n},
\end{aligned}
$$

by Lemma 2.7 (ii). Here $c$ is the constant in (1.4) and $A_{n}$ is finite. Summing up in 
$l$, we get

$$
\lambda\left(r \xi^{0}+y+B_{1}\right) \leqslant c_{4} r^{-(1+\alpha) n} \leqslant c_{1}(1+r)^{-(1+\alpha) n}
$$

with some $c_{4}$ and $c_{1}$.

To get the lower bound, we have only to use the proof of Theorem 1.4 (ii).

The following result is related to the works of Hiraba [12, 13, and Byczkowski et al. 5. Note that $C_{\sigma}=\bigcup_{n=1}^{d} T_{\sigma}(n)$ in the case where $S_{\sigma}$ is a finite set.

Theorem 3.10. Suppose that $S_{\sigma}$ is a finite set. Assume that $\xi^{0} \in T_{\sigma}(n)$ with $1 \leqslant n \leqslant d$. In the case where $0<\alpha<1$ and $\nu$ is one-sided, we assume in addition that $\xi^{0} \in \operatorname{int} C_{\sigma}$. Given $\delta>0$, we can find $c_{1}>0$ and $c_{2}>0$ such that

$$
c_{1}(1+r)^{-(1+\alpha) n} \leqslant p\left(r \xi^{0}+y\right) \leqslant c_{2}(1+r)^{-(1+\alpha) n} \quad \text { whenever }|y| \leqslant \delta .
$$

Proof. Use Lemma 3.9. Then apply Lemma2.5 for a one-point set $K_{1}=K_{2}=\left\{\xi^{0}\right\}$. The lower bound is also obtained by Theorem 1.4 (ii).

Remark 3.11. Let $K$ be a compact set in $T_{\sigma}(n)$. Then the assertion in Theorem 3.10 is true uniformly with respect to $\xi^{0} \in K$. In order to convince ourselves of this, we have only to reexamine the proof of Lemma 3.9 and Remark 2.8.

We conclude this section by considering the two-dimensional case $(d=2)$.

Lemma 3.12. Suppose that $d=2$. Assume that $\sigma=\sum_{j=1}^{N} \sigma_{j}, 1 \leqslant N<\infty$, with $S_{\sigma_{1}}, \ldots, S_{\sigma_{N}}$ being disjoint, each $S_{\sigma_{j}}$ is a closed interval (not a one-point set) in $S^{1}$, and there are $0<a_{j} \leqslant b_{j}<\infty$ such that

$$
a_{j} m(d \xi) \leqslant \sigma_{j}(d \xi) \leqslant b_{j} m(d \xi) \quad \text { on } S_{\sigma_{j}} .
$$

Then, given $\delta_{1}>0$, we can find $c_{1}>0$ and $c_{2}>0$ such that, for any $\xi^{0} \in S_{\sigma}$ (if $1 \leqslant \alpha<2$ or if $0<\alpha<1$ and $\sigma$ is not one-sided) or for any $\xi^{0} \in S_{\sigma} \cap \operatorname{int} C_{\sigma}$ (if $0<\alpha<1$ and $\sigma$ is one-sided),

$$
c_{1}(1+r)^{-(2+\alpha)} \leqslant p\left(r \xi^{0}+y\right) \leqslant c_{2}(1+r)^{-(2+\alpha)} \quad \text { for }|y| \leqslant \delta_{1} .
$$

Moreover, given a compact set $K \subset C_{\sigma}^{0} \backslash S_{\sigma}$ and $\delta_{2}>0$, we can find $c_{3}>0$ and $c_{4}>0$ such that

$$
c_{3}(1+r)^{-(2+2 \alpha)} \leqslant p\left(r \xi^{0}+y\right) \leqslant c_{4}(1+r)^{-(2+2 \alpha)} \quad \text { for } \xi^{0} \in K,|y| \leqslant \delta_{2} .
$$

Proof. The condition (1.26) is satisfied for $U=\operatorname{int} S_{\sigma}$ and $K=S_{\sigma}$. Hence the estimates in (3.22) are consequences of Theorem 1.5. The estimate from below in (3.23) follows from Theorem 1.1 (iii). Let $K$ be a compact set in $C_{\sigma}^{0} \backslash S_{\sigma}$. Note that $C_{\sigma}^{0} \backslash S_{\sigma}=T_{\sigma}(2)$. Thus we see from Theorem 1.4 (i) that the estimate from above in (3.23) is true with $\phi_{1}(r)=r^{-1}$ and $n=2$. The constant $c_{4}$ is taken uniformly for $\xi^{0} \in K$ thanks to Remark 2.8.

It is still hard to give the proper asymptotic order in the case where $\sigma$ is a mixture of an absolutely continuous part and a discrete part. However, we can show them for $d=2$.

Theorem 3.13. Suppose that $d=2$. Assume that there are nonnegative integers $N_{1}$ and $N_{2}$ such that

$$
\sigma(d \xi)=\sum_{k=1}^{N_{1}} c_{k} \delta_{\xi_{k}}+\sum_{j=1}^{N_{2}} \sigma_{j}(d \xi)
$$


where $c_{k}>0$ for all $k, \xi_{1}, \ldots, \xi_{N_{1}}$ are distinct points in $S^{1}, \delta_{\xi_{k}}$ is the $\delta$-measure at $\xi_{k}, \sigma_{j}$ is m-absolutely continuous for each $j, S_{\sigma_{1}}, \ldots, S_{\sigma_{N_{2}}}$ are disjoint closed intervals (not one-point sets) in $S^{1}$, and there are $0<a_{j} \leqslant b_{j}<\infty$ such that

$$
a_{j} m(d \xi) \leqslant \sigma_{j}(d \xi) \leqslant b_{j} m(d \xi) \quad \text { on } S_{\sigma_{j}} .
$$

Further assume that $N_{1} \geqslant 2$ or $N_{2} \geqslant 1$. Let $P_{\sigma}=\left\{\xi_{1}, \ldots, \xi_{N_{1}}\right\}$. Let $\xi^{0} \in S^{d-1}$. If $0<\alpha<1$ and $\sigma$ is one-sided, we assume that $\xi^{0} \in \operatorname{int} C_{\sigma}$. Let $\delta>0$. Note that $C_{\sigma}=P_{\sigma} \cup\left(S_{\sigma} \backslash P_{\sigma}\right) \cup\left(C_{\sigma}^{0} \backslash S_{\sigma}\right)$. If $\xi^{0} \in P_{\sigma}$, then we can find $c_{1}>0$ and $c_{2}>0$ such that

$$
c_{1}(1+r)^{-(1+\alpha)} \leqslant p\left(r \xi^{0}+y\right) \leqslant c_{2}(1+r)^{-(1+\alpha)} \quad \text { for }|y| \leqslant \delta .
$$

If $\xi^{0} \in S_{\sigma} \backslash P_{\sigma}$, then we can find $c_{3}>0$ and $c_{4}>0$ such that

$$
c_{3}(1+r)^{-(2+\alpha)} \leqslant p\left(r \xi^{0}+y\right) \leqslant c_{4}(1+r)^{-(2+\alpha)} \quad \text { for }|y| \leqslant \delta .
$$

If $\xi^{0} \in C_{\sigma}^{0} \backslash S_{\sigma}$, then we can find $c_{5}>0$ and $c_{6}>0$ such that

$$
c_{5}(1+r)^{-(2+2 \alpha)} \leqslant p\left(r \xi^{0}+y\right) \leqslant c_{6}(1+r)^{-(2+2 \alpha)} \quad \text { for }|y| \leqslant \delta .
$$

Proof. Hereafter $c_{1}, c_{2}, \ldots$ are positive constants. For $\xi^{0} \in P_{\sigma}$, (3.26) is true by Corollary 3.4 .

Assume $N_{1} \geqslant 2$ and $N_{2} \geqslant 1$. Let $\xi^{0} \in S_{\sigma} \backslash P_{\sigma}$ and $|y| \leqslant \delta$. Let us prove (3.27). Denote the first and the second term in the right-hand side of (3.24) by $\sigma^{\mathrm{d}}$ and $\sigma^{\mathrm{ac}}$, respectively. Let $\left\{X_{t}^{\mathrm{d}}\right\}$ and $\left\{X_{t}^{\mathrm{ac}}\right\}$ be independent $\alpha$-stable processes such that $\left\{X_{t}\right\} \stackrel{\mathrm{d}}{=}\left\{X_{t}^{\mathrm{d}}+X_{t}^{\mathrm{ac}}\right\}$ and the $\sigma$-measures of $\left\{X_{t}^{\mathrm{d}}\right\}$ and $\left\{X_{t}^{\mathrm{ac}}\right\}$ are equal to $\sigma^{\mathrm{d}}$ and $\sigma^{\mathrm{ac}}$, respectively. They are nondegenerate. Let $p^{\mathrm{d}}(x)$ and $p^{\text {ac }}(x)$ be their continuous densities at time 1 . Then

$$
p\left(r \xi^{0}+y\right)=\int_{\mathbb{R}^{2}} p^{\mathrm{ac}}\left(r \xi^{0}+y-z\right) p^{\mathrm{d}}(z) d z .
$$

In the same way, we decompose $\left\{Z_{t}\right\}$ as $\left\{Z_{t}\right\} \stackrel{\mathrm{d}}{=}\left\{Z_{t}^{\mathrm{d}}+Z_{t}^{\text {ac }}\right\}$. Let $\lambda^{\mathrm{d}}$ and $\lambda^{\text {ac }}$ be the distribution of $Z_{1}^{\mathrm{d}}$ and $Z_{1}^{\mathrm{ac}}$. Then, as in the proof of Theorem 1.5 (ii), there is $R_{1}>0$ such that

$$
\lambda\left(r \xi^{0}+B_{1}\right) \geqslant c_{1} \lambda^{\mathrm{ac}}\left(r \xi^{0}+B_{1}\right) \geqslant c_{2}(1+r)^{-(2+\alpha)} \quad \text { for } r>R_{1} .
$$

This gives the lower estimate by Lemma 2.5 (ii) with $K_{2}=\left\{\xi^{0}\right\}$. Next, let us show the upper estimate. When $\xi^{0} \in T_{\sigma^{\mathrm{d}}}(2)$, choose a small $0<\delta_{1}<1$ such that if $\left|r \xi^{0}-z\right| \leqslant \delta_{1} r$, then $z /|z| \in T_{\sigma^{\mathrm{d}}}(2)$. This is possible as in the proof of Lemma 2.7. When $\xi^{0} \notin T_{\sigma^{\mathrm{d}}}(2)$, choose $0<\delta_{1}<1$ such that if $\left|r \xi^{0}-z\right| \leqslant \delta_{1} r$, then $z /|z| \notin C_{\sigma^{\mathrm{d}}}$ (note that $\xi^{0} \notin C_{\sigma^{\mathrm{d}}}$ since $C_{\sigma^{\mathrm{d}}} \backslash C_{\sigma^{\mathrm{d}}}^{0} \subset S_{\sigma^{\mathrm{d}}}=P_{\sigma}$ ). Write (3.29) as $p\left(r \xi^{0}+y\right)=I_{1}+I_{2}$, where

$I_{1}=\int_{\left|r \xi^{0}-z\right| \leqslant \delta_{1} r} p^{\mathrm{ac}}\left(r \xi^{0}+y-z\right) p^{\mathrm{d}}(z) d z, \quad I_{2}=\int_{\left|r \xi^{0}-z\right|>\delta_{1} r} p^{\mathrm{ac}}\left(r \xi^{0}+y-z\right) p^{\mathrm{d}}(z) d z$.

If $\xi^{0} \in T_{\sigma^{\mathrm{d}}}(2)$, then

$$
p^{\mathrm{d}}(z) \leqslant c_{3}(1+|z|)^{-2(1+\alpha)} \leqslant c_{4}(1+r)^{-2(1+\alpha)}
$$

for $\left|r \xi^{0}-z\right| \leqslant \delta_{1} r$ and for large $r$, by Theorem 3.10 and Remark 3.11 , If $\xi^{0} \notin T_{\sigma^{\mathrm{d}}}(2)$, then

$$
p^{\mathrm{d}}(z) \leqslant c_{5} e^{-|z| \log |z|} \leqslant c_{6} e^{-r \log r}
$$


for $\left|r \xi^{0}-z\right| \leqslant \delta_{1} r$, by Theorem 1.1 (iv). Thus we have $I_{1} \leqslant c_{7}(1+r)^{-2(1+\alpha)}$. On the other hand, $I_{2} \leqslant c_{8}(1+r)^{-(2+\alpha)}$, since

$$
p^{\mathrm{ac}}\left(r \xi^{0}+y-z\right) \leqslant c_{9}(1+r)^{-(2+\alpha)}
$$

for $\left|r \xi^{0}-z\right|>\delta_{1} r$ with large $r$, by Theorem 1.5 (i). Thus we get (3.27).

Still keeping the assumption that $N_{1} \geqslant 2$ and $N_{2} \geqslant 1$, let us next prove (3.28) for $\xi^{0} \in C_{\sigma}^{0} \backslash S_{\sigma}$. The lower estimate follows from Theorem 1.1 (iii). To get the upper estimate, let $I_{1}, I_{2}$, and $I_{3}$ be the integral in (3.29) with domain of integration changed to $\left\{z:\left|r \xi^{0}-z\right| \leqslant \delta_{1} r\right\},\left\{z:\left|r \xi^{0}-z\right|>\delta_{1} r\right.$ and $\left.|z| \leqslant \delta_{2} r\right\}$, and $\left\{z:\left|r \xi^{0}-z\right|>\delta_{1} r\right.$ and $\left.|z|>\delta_{2} r\right\}$, respectively, where $\delta_{1}>0$ and $\delta_{2}>0$ are chosen sufficiently small. Then we can check $I_{j} \leqslant c_{j}(1+r)^{-(2+2 \alpha)}$ for $j=1,2,3$. Indeed, for $j=1$ this is by the same discussion as in the case where $\xi^{0} \in S_{\sigma} \backslash P_{\sigma}$. For $j=2$, we consider two cases, $\xi^{0} \in C_{\sigma^{\text {ac }}}^{0}$ and $\xi^{0} \notin C_{\sigma^{\mathrm{ac}}}^{0}$. In the first case we can use

$$
p^{\mathrm{ac}}\left(r \xi^{0}+y-z\right) \leqslant c_{4}\left(1+\left|r \xi^{0}-z\right|\right)^{-(2+2 \alpha)} \leqslant c_{5}(1+r)^{-(2+2 \alpha)}
$$

for large $r$ by Lemma 3.12, letting $\Pi\left(r \xi^{0}-z\right) \in C_{\sigma^{\text {ac }}}^{0} \backslash S_{\sigma^{\text {ac }}}$. In the second case we can use Theorem 1.1 (iv). For $j=3$, notice that

$$
p^{\mathrm{ac}}\left(r \xi^{0}+y-z\right) \leqslant c_{6}\left(1+\left|r \xi^{0}-z\right|\right)^{-(2+\alpha)} \leqslant c_{7}(1+r)^{-(2+\alpha)}
$$

for large $r$ by Theorem 1.5 (i), and that

$$
P\left(\left|W_{1}\right|>s\right) \leqslant \operatorname{const}(1+s)^{-\alpha} \quad \text { for } s \geqslant 0
$$

for any $\alpha$-stable process $\left\{W_{t}\right\}$ on $\mathbb{R}^{d}$, to conclude

$$
I_{3} \leqslant c_{7}(1+r)^{-(2+\alpha)} \int_{|z|>\delta_{2} r} p^{\mathrm{d}}(z) d z \leqslant c_{3}(1+r)^{-(2+2 \alpha)} .
$$

The estimate 3.30 is well-known in one dimension and, for general $d$, can be reduced to a one-dimensional case by considering components.

It remains to prove (3.27) and (3.28) in the following three cases: (1) $N_{1} \geqslant 2$ and $N_{2}=0,(2) N_{1}=1$ and $N_{2} \geqslant 1,(3) N_{1}=0$ and $N_{2} \geqslant 1$. Case (1) is treated by Theorem 3.10. The case (3) is done by Lemma 3.12, In case (2), with the choice of $\tau^{\mathrm{d}}=0$, there is a straight line $V$ through the origin such that the distribution $\rho$ of $X_{1}^{\mathrm{d}}$ is concentrated on $V$, thus $p\left(r \xi^{0}+y\right)=\int_{V} p^{\mathrm{ac}}\left(r \xi^{0}+y-z\right) \rho(d z)$, and we note that $\operatorname{dis}\left(r \xi^{0}, V\right)=c_{1} r$ with some $c_{1}>0$ if $\xi^{0} \notin P_{\sigma}$. Thus the proof is similar to the case of $N_{1} \geqslant 2$ and $N_{2} \geqslant 1$.

\section{Applications to stable processes}

We study implications of our results on the asymptotic estimates in the set $\mathfrak{T}$ below and in the Spitzer type limit theorems mentioned in the last paragraph of Section 1. Let $X=\left(\Omega, \mathcal{F}, \mathcal{F}_{t}, X_{t}, \theta_{t}, P^{x}\right)$ be the standard (Markov) process in the sense of Blumenthal and Getoor [4] induced by a Lévy process $\left\{X_{t}\right\}$ on $\mathbb{R}^{d}$. That is, $P^{x}\left(X_{t} \in B\right)=P\left(x+X_{t} \in B\right)$ for any $x \in \mathbb{R}^{d}, t \geqslant 0$, and Borel set $B$. The symbol $E^{x}$ stands for the expectation under $P^{x}$. Denote the dual process of $X$ by $\widetilde{X}=\left(\Omega, \mathcal{F}, \mathcal{F}_{t}, X_{t}, \theta_{t}, \widetilde{P}^{x}\right)$. Let $T_{B}$ be the hitting time of a Borel set $B$ defined by $T_{B}=\inf \left\{t>0: X_{t} \in B\right\}$, where we understand $T_{B}=\infty$ if $X_{t} \notin B$ for all $t>0$. Let $L_{B}$ be the last exit time from an open set $B$, that is, $L_{B}=\sup \left\{t \geqslant 0: X_{t} \in B\right\}$. Let $B_{r}=\{x:|x|<r\}$. The process is recurrent if and only if $L_{B_{r}}=\infty$ a.s. for all $r>0$; it is transient if and only if $L_{B_{r}}<\infty$ a.s. for all $r>0$. Recall that, in 
general, $A(t) \asymp B(t)$ means that there are constants $0<c_{1} \leqslant c_{2}<\infty$ such that $c_{1} B(t) \leqslant A(t) \leqslant c_{2} B(t)$ for all sufficiently large $t$.

Lemma 4.1. Let $\left\{X_{t}\right\}$ be a transient Lévy process on $\mathbb{R}^{d}$. Let $\eta>0$. Then one of the following is true:

$$
\begin{array}{ll}
E^{0}\left[L_{B_{r}}{ }^{\eta}\right]<\infty & \text { for all } r>0 \\
E^{0}\left[L_{B_{r}}{ }^{\eta}\right]=\infty & \text { for all } r>0 .
\end{array}
$$

This is Theorem 2.8 of [33]. Given a transient Lévy process on $\mathbb{R}^{d}$, denote

$$
\mathfrak{T}=\{0\} \cup\{\eta>0: \text { (4.1) is true }\} .
$$

The bigger this set $\mathfrak{T}$, the stronger a degree of transience. The process $\left\{X_{t}\right\}$ is called strongly transient if $1 \in \mathfrak{T}$; it is called weakly transient if $1 \notin \mathfrak{T}$. Hawkes [11] and Takeuchi [37] are early works on the moment of the last exit time for a symmetric Lévy process. Strong transience is important in the theory of the range of random walks. See Jain and Pruitt [15]. After Sato [29], 30], the set $\mathfrak{T}$ has been studied in detail by Sato and Watanabe [33, 34. In [33, they obtained a criterion for $\eta \in \mathfrak{T}$ for a general transient Lévy process and discussed the relation with the moment of the last exit time from a half line for a one-dimensional random walk. This had been studied by Janson [16] and Kesten and Maller [18. Dawson et al. 6] investigated the application of the set $\mathfrak{T}$ in branching systems. The following is Lemma 2.3 of 33 .

Lemma 4.2. Let $\left\{X_{t}\right\}$ be a transient Lévy process on $\mathbb{R}^{d}$. Let $\eta>0$. Then $\eta \in \mathfrak{T}$ if and only if

$$
\int_{0}^{\infty} t^{\eta} P^{0}\left[X_{t} \in B_{1}\right] d t<\infty
$$

$\eta \notin \mathfrak{T}$ if and only if

$$
\int_{0}^{\infty} t^{\eta} P^{0}\left[X_{t} \in B_{1}\right] d t=\infty
$$

Let us remark the following fact (32] Theorems 37.8, 37.16, 37.18).

Lemma 4.3. Let $\left\{X_{t}\right\}$ be a nondegenerate $\alpha$-stable process on $\mathbb{R}^{d}$. If $d \geqslant 3$, then it is transient. If $d=1$ or 2 , then it is recurrent if and only if it is first-class $\alpha$-stable with $d \leqslant \alpha \leqslant 2$.

Define $E_{B}(t)$ for $t \geqslant 0$ as

$$
E_{B}(t)=\int_{\mathbb{R}^{d}} P^{x}\left(T_{B} \leqslant t\right) d x
$$

Asymptotic expansion of $E_{B}(t)$ as $t \rightarrow \infty$ for a bounded Borel set $B$ has been a subject of research in many papers since Spitzer [36] in 1964 for a transient Brownian motion. Later Le Gall [19] extended Spitzer's results by determining the more exact order of the asymptotic expansion conjectured by M. Kac in a footnote of Spitzer [36. Assume that the original Lévy process $\left\{X_{t}\right\}$ on $\mathbb{R}^{d}$ is transient and nondegenerate. Let $C(B)$ be the capacity for a Borel set $B$. We have $0 \leqslant C(B)<\infty$ for any bounded Borel set B. See Port and Stone [26, Sato [32, Chapter 8, and 
Bertoin 2, Chapter 2. Fix a nonnegative continuous function $f(x)$ with compact support, $f(0)>0$ and $\int_{\mathbb{R}^{d}} f(x) d x=1$. Let

$$
r(t)=\int_{t}^{\infty} d s \int_{\mathbb{R}^{d}} f(x)\left(E^{x} f\left(X_{s}\right)\right) d x .
$$

We have $r(t)<\infty$ for $t \geqslant 0$ because of the transience. In the following, let $B$ be a bounded Borel set in $\mathbb{R}^{d}$. In the case where $X_{t}$ has a purely singular distribution for every $t>0$, we make an additional assumption that $P^{x}\left(T_{\bar{B}}=T_{\operatorname{int} B}\right)=1$ for almost every $x$. Let

$$
\varphi_{B}(x)=P^{x}\left(T_{B}<\infty\right), \quad \widetilde{\varphi}_{B}(x)=\widetilde{P}^{x}\left(T_{B}<\infty\right) .
$$

If $1 \in \mathfrak{T}$, then $\int_{\mathbb{R}^{d}} \widetilde{\varphi}_{B}(x) \varphi_{B}(x) d x<\infty$. Define

$$
\Delta_{B}^{(1)}(t)=E_{B}(t)-t C(B)
$$

and

$$
\Delta_{B}^{(2)}(t)=E_{B}(t)-t C(B)-\int_{\mathbb{R}^{d}} \widetilde{\varphi}_{B}(x) \varphi_{B}(x) d x .
$$

The following lemma is a result obtained by a series of works [36, 8, [23, 24, 25], 26]. Assertion (i) is given in Theorem 14.2 of [26] and assertion (ii) is in Lemmas 3.1 and 3.3 and Theorem 1 of [25] with (3.19) and (14.13) of [26], respectively. See also Proposition 6.2 of 34 .

Lemma 4.4. (i) If $1 \notin \mathfrak{T}$, then

$$
\Delta_{B}^{(1)}(t)=(C(B))^{2} \int_{0}^{t} r(s) d s+o\left(\int_{0}^{t} r(s) d s\right), \quad t \rightarrow \infty .
$$

(ii) If $1 \in \mathfrak{T}$ and

$$
r(\cdot) \in \mathcal{D}, \quad \text { that is, } \quad \sup _{t>0} \frac{r(t / 2)}{r(t)}<\infty
$$

then

$$
\Delta_{B}^{(2)}(t)=-(C(B))^{2} \int_{t}^{\infty} r(s) d s+o\left(\int_{t}^{\infty} r(s) d s\right), \quad t \rightarrow \infty .
$$

Sato and Watanabe 34 obtained the set $\mathfrak{T}$ for all transient $\alpha$-semistable processes with $d=1$ and classified all transient $\alpha$-semistable processes into weakly transient and strongly transient. Port 23, 24] completely studied the asymptotic order of $\Delta_{B}^{(1)}(t)$ when $1 \notin \mathfrak{T}$ and partially did that of $\Delta_{B}^{(2)}(t)$ when $1 \in \mathfrak{T}$ for transient $\alpha$-stable processes. See also Sato and Watanabe [34 for $\alpha$-semistable processes. Now the case in $1 \in \mathfrak{T}$ where we do not have a proper order of $\Delta_{B}^{(2)}(t)$ is the same as for the set $\mathfrak{T}$, that is, $d \geqslant 2,1 \leqslant \alpha<2$ and second-class with $\sigma(\{-\tau /|\tau|\})=0$. Combining the following lemma and our estimates in Sections 1 and 3 , we can find that the set $\mathfrak{T}$ and the proper asymptotic order of $\Delta_{B}^{(2)}(t)$ as $t \rightarrow \infty$ delicately depend on the direction of $-\tau /|\tau|$ in relation to the location of $S_{\sigma}$ in this case.

Lemma 4.5. Let $\left\{X_{t}\right\}$ be a nondegenerate transient second-class $\alpha$-stable process on $\mathbb{R}^{d}$ with $1 \leqslant \alpha<2$ and $\sigma(\{-\tau /|\tau|\})=0$. Assume that $d \geqslant 2$ and that there are $\beta \geqslant 1+\alpha, c_{1}>0, c_{2}>0$ and $R>0$ such that

$$
c_{1}(1+r)^{-\beta} \leqslant p(-r \tau+y) \leqslant c_{2}(1+r)^{-\beta} \quad \text { for } r \geqslant R,|y| \leqslant 1 .
$$


(i) If $1<\alpha<2$, then

$$
\mathfrak{T}=[0, d / \alpha+(1-1 / \alpha) \beta-1) \quad \text { and } \quad-\Delta_{B}^{(2)}(t) \asymp t^{2-d / \alpha-(1-1 / \alpha) \beta} .
$$

(ii) If $\alpha=1$, then

$$
\mathfrak{T}=[0, d-1] \quad \text { and } \quad-\Delta_{B}^{(2)}(t) \asymp \begin{cases}(\log t)^{-\beta+1} & \text { for } d=2, \\ t^{2-d}(\log t)^{-\beta} & \text { for } d \geqslant 3 .\end{cases}
$$

Proof. For any $\eta>0$, it follows from Lemma 4.2 that $\int_{0}^{\infty} t^{\eta-1} r(t) d t<\infty$ if and only if $\eta \in \mathfrak{T}$. We prove (i). Suppose that $1<\alpha<2$. We have by $(2.3)$

$$
r(t) \asymp \int_{|x| \leqslant 1} d x \int_{t}^{\infty} p(s, x) d s \asymp t^{1-d / \alpha-(1-1 / \alpha) \beta}, \quad t \rightarrow \infty,
$$

and hence $\mathfrak{T}=[0, d / \alpha+(1-1 / \alpha) \beta-1)$ and

$$
\int_{t}^{\infty} r(s) d s \asymp t^{2-d / \alpha-(1-1 / \alpha) \beta}, \quad t \rightarrow \infty .
$$

Now use Lemma 4.4. Next we prove (ii). Suppose that $\alpha=1$. We have by (2.3)

$$
r(t) \asymp \int_{|x| \leqslant 1} d x \int_{t}^{\infty} p(s, x) d s \asymp(\log t)^{-\beta} t^{-(d-1)}, \quad t \rightarrow \infty .
$$

Hence $\mathfrak{T}=[0, d-1]$ and

$$
\int_{t}^{\infty} r(s) d s \asymp \begin{cases}(\log t)^{-\beta+1} & \text { for } d=2, \\ (\log t)^{-\beta} t^{-(d-2)} & \text { for } d \geqslant 3 .\end{cases}
$$

Then use Lemma 4.4.

In the following propositions, assume $d \geqslant 2,1 \leqslant \alpha<2$ and that our stable process $\left\{X_{t}\right\}$ is second-class.

Proposition 4.6. Suppose that the assumptions in Theorem 3.8 are satisfied for our process $\left\{X_{t}\right\}$.

(i) If $1<\alpha<2$, and $-\tau /|\tau| \in T(U, n)$ with $1 \leqslant n \leqslant d$, then

$$
\mathfrak{T}=[0, d+n(\alpha-1)-1) \quad \text { and } \quad-\Delta_{B}^{(2)}(t) \asymp t^{2-d-n(\alpha-1)} .
$$

(ii) If $\alpha=1$, and $-\tau /|\tau| \in T(U, n)$ with $1 \leqslant n \leqslant d$, then

$$
\mathfrak{T}=[0, d-1] \quad \text { and } \quad-\Delta_{B}^{(2)}(t) \asymp \begin{cases}(\log t)^{-1-n} & \text { for } d=2, \\ t^{2-d}(\log t)^{-d-n} & \text { for } d \geqslant 3 .\end{cases}
$$

Proposition 4.7. Assume that $S_{\sigma}$ is a finite set. By Theorem 3.10, we have the following:

(i) If $1<\alpha<2$ and $-\tau /|\tau| \in T_{\sigma}(n)$ with $1 \leqslant n \leqslant d$, then

$$
\mathfrak{T}=[0, d / \alpha+(\alpha-1 / \alpha) n-1) \quad \text { and } \quad-\Delta_{B}^{(2)}(t) \asymp t^{2-d / \alpha-(\alpha-1 / \alpha) n} .
$$

(ii) If $\alpha=1$ and $-\tau /|\tau| \in T_{\sigma}(n)$ with $1 \leqslant n \leqslant d$, then

$$
\mathfrak{T}=[0, d-1] \quad \text { and } \quad-\Delta_{B}^{(2)}(t) \asymp \begin{cases}(\log t)^{-2 n+1} & \text { for } d=2, \\ t^{2-d}(\log t)^{-2 n} & \text { for } d \geqslant 3 .\end{cases}
$$


Proposition 4.8. Suppose that the assumptions in Theorem 3.13 are satisfied for our process $\left\{X_{t}\right\}$ with $d=2$. Note that $C_{\sigma}^{0} \backslash S_{\sigma}=C_{\sigma} \backslash S_{\sigma}$.

(i) Assume $1<\alpha<2$.

(1) If $-\tau /|\tau| \in P_{\sigma}$, then

$$
\mathfrak{T}=[0, \alpha+1 / \alpha-1) \quad \text { and } \quad-\Delta_{B}^{(2)}(t) \asymp t^{2-\alpha-1 / \alpha} .
$$

(2) If $-\tau /|\tau| \in S_{\sigma} \backslash P_{\sigma}$, then

$$
\mathfrak{T}=[0, \alpha) \quad \text { and } \quad-\Delta_{B}^{(2)}(t) \asymp t^{1-\alpha} .
$$

(3) If $-\tau /|\tau| \in C_{\sigma}^{0} \backslash S_{\sigma}$, then

$$
\mathfrak{T}=[0,2 \alpha-1) \quad \text { and } \quad-\Delta_{B}^{(2)}(t) \asymp t^{2-2 \alpha} .
$$

(ii) Assume $\alpha=1$.

(1) If $-\tau /|\tau| \in P_{\sigma}$, then

$$
\mathfrak{T}=[0,1] \quad \text { and } \quad-\Delta_{B}^{(2)}(t) \asymp(\log t)^{-1} .
$$

(2) If $-\tau /|\tau| \in S_{\sigma} \backslash P_{\sigma}$, then

$$
\mathfrak{T}=[0,1] \quad \text { and } \quad-\Delta_{B}^{(2)}(t) \asymp(\log t)^{-2} .
$$

(3) If $-\tau /|\tau| \in C_{\sigma}^{0} \backslash S_{\sigma}$, then

$$
\mathfrak{T}=[0,1] \quad \text { and } \quad-\Delta_{B}^{(2)}(t) \asymp(\log t)^{-3} .
$$

The final proposition shows that the asymptotics of $\Delta_{B}^{(2)}(t)$ can be more delicate than those of Lemma 4.5 in a certain special case.

Proposition 4.9. Let $\ell(r)$ and $\ell^{*}(r)$ be slowly varying as in Remark 3.3. Suppose that $d \geqslant 2,1 \leqslant \alpha<2$ and second-class, and further $\xi^{0}=-\tau /|\tau|$ satisfies (3.4) and (3.5) with $\sigma_{\xi^{0}}(r) \asymp r^{-s} \ell(r)$ and $0<s<d-1$ or with $\sigma_{\xi^{0}}(r) \asymp \ell^{*}(r)$.

(i) Assume $1<\alpha<2$. Then $\eta \in \mathfrak{T}$ if and only if $\int_{1}^{\infty} t^{\eta-(d-1) / \alpha-\alpha} \sigma_{\xi^{0}}\left(t^{1-1 / \alpha}\right) d t$ $<\infty$.

(1) If $\sigma_{\xi^{0}}(r) \asymp r^{-s} \ell(r)$ with $0<s<d-1$, then

$$
-\Delta_{B}^{(2)}(t) \asymp t^{2-(d-1) / \alpha-\alpha-(1-1 / \alpha) s} \ell\left(t^{1-1 / \alpha}\right) .
$$

(2) If $\sigma_{\xi^{0}}(r) \asymp \ell^{*}(r)$, then

$$
-\Delta_{B}^{(2)}(t) \asymp t^{2-(d-1) / \alpha-\alpha} \ell^{*}\left(t^{1-1 / \alpha}\right) .
$$

(ii) Assume $\alpha=1$. Then $\mathfrak{T}=[0, d-1]$.

(1) If $\sigma_{\xi^{0}}(r) \asymp r^{-s} \ell(r)$ with $0<s<d-1$, then

$$
-\Delta_{B}^{(2)}(t) \asymp \begin{cases}(\log t)^{-1-s} \ell(\log t) & \text { for } d=2, \\ t^{2-d}(\log t)^{-2-s} \ell(\log t) & \text { for } d \geqslant 3 .\end{cases}
$$

(2) If $\sigma_{\xi^{0}}(r) \asymp \ell^{*}(r)$, then

$$
-\Delta_{B}^{(2)}(t) \asymp \begin{cases}(\log t)^{-1} \ell^{*}(\log t) & \text { for } d=2, \\ t^{2-d}(\log t)^{-2} \ell^{*}(\log t) & \text { for } d \geqslant 3 .\end{cases}
$$

Proof. Proof is similar to Lemma 4.5 by using (2.3). 
We add some final remarks on the set $\mathfrak{T}$.

Remark 4.10. Let $\left\{X_{t}\right\}$ be a nondegenerate second-class transient $\alpha$-stable process with $d \geqslant 2$ and $1 \leqslant \alpha<2$.

(i) Suppose that $-\tau /|\tau| \in C_{\sigma}^{0}$. Then $\mathfrak{T} \subset[0, d \alpha-1)$ for $1<\alpha<2$ and $\mathfrak{T}=$ $[0, d-1]$ for $\alpha=1$.

(ii) Suppose that there is $\phi \in \mathcal{D}$ such that

$$
\liminf _{r \rightarrow \infty} \frac{\sigma\left(\xi+B_{1 / r}\right)}{\phi(r)}>0 \quad \text { for any } \xi \in S_{\sigma} .
$$

If $-\tau /|\tau| \in \bigcup_{n=1}^{d} T_{\sigma}(n)$, then $\mathfrak{T}=[0, d-1]$ for $\alpha=1$ and $\mathfrak{T}$ is bounded for $1<\alpha<2$.

(iii) If $-\tau /|\tau| \notin C_{\sigma}$, then $\mathfrak{T}=[0, \infty)$.

Proof. (i) If $-\tau /|\tau| \in C_{\sigma}^{0}$ for $1<\alpha<2$, then we see from (iii) of Theorem 1.1 that, for $\eta \geqslant d \alpha-1$,

$$
\int_{|x| \leqslant 1} d x \int_{1}^{\infty} t^{\eta} p(t, x) d t \geqslant c_{1} \int_{1}^{\infty} t^{\eta-d / \alpha-(1-1 / \alpha) d(1+\alpha)} d t=\infty
$$

with some $c_{1}>0$. Thus $\mathfrak{T} \subset[0, d \alpha-1)$ for $1<\alpha<2$ by Lemma 4.2. The proof of $\mathfrak{T}=[0, d-1]$ for $\alpha=1$ is similar by using Theorem B (i) of 34 .

(ii) Let $\alpha=1$ and $-\tau /|\tau| \in \bigcup_{n=1}^{d} T_{\sigma}(n)$. By Theorem B (i) of [34, we have $\mathfrak{T} \supset[0, d-1]$. Since $\phi \in \mathcal{D}$, there are $B \geqslant 0$ and $c_{2}>0$ such that

$$
\phi(1+r) \geqslant c_{2}(1+r)^{-B} \quad \text { for } r \geqslant 0 .
$$

By Theorem 1.4 (ii) we have, for $\eta>d-1$,

$$
\begin{gathered}
\int_{|x| \leqslant 1} d x \int_{1}^{\infty} t^{\eta} p(t, x) d t=\int_{|x| \leqslant 1} d x \int_{1}^{\infty} t^{\eta-d} p\left(t^{-1} x-(\log t) \tau\right) d t \\
\geqslant c_{3} \int_{1}^{\infty} t^{\eta-d}(\log t)^{-d(2+B)} d t=\infty
\end{gathered}
$$

with some $c_{3}>0$. Hence $\mathfrak{T} \subset[0, d-1]$ by Lemma 4.2. Let $1<\alpha<2$ and $-\tau /|\tau| \in \cup_{n=1}^{d} T_{\sigma}(n)$. Then we see that $\mathfrak{T} \subset[0, \alpha d+(1-1 / \alpha) B d-1)$ as above.

The proof of (iii) is similar by using (iv) of Theorem 1.1.

The complete study of determining the set $\mathfrak{T}$ and the proper order of $\Delta_{B}^{(2)}(t)$ for the transient stable process is still far from us. The situation is more difficult for transient semistable processes. A result of Ishikawa [14 suggests that there might be an essential difference of the asymptotics of $\Delta_{B}^{(2)}(t)$ between stable and semistable processes. We finish this article by posing a natural conjecture related to Proposition 6.3 of [34]. Let $\left\{X_{t}\right\}$ be a transient Lévy process. Define two indices $a_{1}$ for $1 \notin \mathfrak{T}$ and $a_{2}$ for $1 \in \mathfrak{T}$ as

$$
a_{1}=\inf \left\{c \geqslant 0: \limsup _{t \rightarrow \infty} \frac{\Delta_{B}^{(1)}(t)}{t^{c}}<\infty\right\}
$$

and

$$
a_{2}=\sup \left\{c \geqslant 0: \limsup _{t \rightarrow \infty} \frac{-\Delta_{B}^{(2)}(t)}{t^{-c}}<\infty\right\} .
$$

Then we conjecture that if $\overline{\mathfrak{T}}=[0, b]$ with $0 \leqslant b<\infty$, then $a_{1}=1-b$ for $1 \notin \mathfrak{T}$ and $a_{2}=b-1$ for $1 \in \mathfrak{T}$. 


\section{ACKNOWLEDGEMENTS}

The author would like to thank K. Sato for his valuable advice to the early draft and helpful discussions. He is also grateful to a referee for careful reading and pointing out to him [22].

\section{REFERENCES}

[1] S. V. Arkhipov, The density function's asymptotic representation in the case of multidimensional strictly stable distributions, Lecture Notes in Math., 1412 (1987), 1-21, SpringerVerlag, Berlin. MR.1041341 (91e:60043)

[2] J. Bertoin, Lévy Processes, Cambridge Univ. Press, Cambridge, 1996. MR 1406564 (98e:60117)

[3] N. H. Bingham, C.M. Goldie and J.L. Teugels, Regular Variation, Cambridge University Press, Cambridge, 1987. MR0898871 (88i:26004)

[4] R. M. Blumenthal and R.K. Getoor, Markov Processes and Potential Theory, Academic Press, New York, 1968. MR0264757 (41:9348)

[5] T. Byczkowski, J.P. Nolan and B. Rajput, Approximation of multidimensional stable densities, J. Multivariate Anal. 46 (1993), 13-31. MR.1231241 (94f:60023)

[6] D. A. Dawson, L.G. Gorostiza and A. Wakolbinger, Occupation time fluctuations in branching systems, J. Theoret. Probab. 14 (2001), 729-796. MR:1860521(2002h:60183)

[7] J. Dziubański, Asymptotic behaviour of densities of stable semigroups of measures, Probab. Th. Rel. Fields 87 (1991), 459-467. MR1085177(92a:60025)

[8] R. K. Getoor, Some asymptotic formulas involving capacity, Zeit. Wahrsch. Verw. Gebiete 4 (1965), 248-252. MR0190988 (32:8397)

[9] P. Głowacki, Lipschitz continuity of densities of stable semigroups of measures, Colloq. Math. 66 (1993), 29-47. MR1242643 (94k:43008)

[10] P. Głowacki and W. Hebisch, Pointwise estimates for densities of stable semigroups of measures, Studia Math. 104 (1993), 243-258. MR1220664 (95e:22016)

[11] J. Hawkes, Moments of last exit times, Mathematika 24 (1977), 266-269. MR0488316 $(58: 7865)$

[12] S. Hiraba, Asymptotic behaviour of densities of multi-dimensional stable distributions, Tsukuba J. Math. 18 (1994), 223-246. MR.1287843 (95g:60024)

[13] S. Hiraba, Asymptotic estimates for densities of multi-dimensional stable distributions, Tsukuba J. Math. 27 (2003), 261-287. MR2025727(2004i:62081)

[14] Y. Ishikawa, Density estimate in small time for jump processes with singular Lévy measures. Tohoku Math. J. 53 (2001), 183-202. MR.1829978 (2002g:60124)

[15] N. Jain and W.E. Pruitt, The range of random walk, Proc. Sixth. Berkeley Symp. Math. Statist. Probab., Vol. 3, Univ. Calif. Press, Berkeley and Los Angeles, 1972, pp. 31-50. MR0410936 (53:14677)

[16] S. Janson, Moments for first-passage and last-exit times, the minimum, and related quantities for random walks with positive drift, Adv. Appl. Probab. 18 (1986), 865-879. MR0867090 (88b:60164)

[17] M. Kanter, Unimodality and dominance for symmetric random vectors, Trans. Amer. Math. Soc. 229 (1977), 65-85. MR0445580 (56:3917)

[18] H. Kesten and R.A.Maller, Two renewal theorems for general random walks tending to infinity, Probab. Th. Rel. Fields 106 (1996), 1-38. MR1408415(97i:60113)

[19] J.-F. Le Gall, Sur une conjecture de M. Kac, Probab. Th. Rel. Fields 78 (1988), 389-402. MR0949180 (89m:60195)

[20] P. Lévy, Théorie de l'Addition des Variables Aléatoires, Gauthier-Villars, Paris, 1937 (2éd., 1954).

[21] P. Mattila, Geometry of Sets and Measures in Euclidean Spaces Fractals and rectifiability, Cambridge Univ. Press, Cambridge, 1995. MR1333890 (96h:28006)

[22] J. Picard, Density in small time at accessible points for jump processes, Stochastic Process Appl. 67 (1997), 251-279. MR1449834(98f:60163)

[23] S. C. Port, Stable processes with drift on the line, Trans. Amer. Math. Soc. 313 (1989), 805-841. MR0997680 (91c:60096) 
[24] S. C. Port, Asymptotic expansions for the expected volume of a stable sausage, Ann. Probab. 18 (1990), 492-523. MR1055417(91h:60084)

[25] S. C. Port, Spitzer's formula involving capacity, Random Walks, Brownian Motion, and Interacting Particle Systems. A Festschrift in Honor of Frank Spitzer (ed. R. Durrett and H. Kesten), Birkhäuser, Boston, 1991, pp. 373-388. MR1146459 (93a:60112)

[26] S. C. Port and C. J. Stone, Infinitely divisible processes and their potential theory, I and II, Ann. Inst. Fourier (Grenoble) 21 (1971), Fasc. 2, 157-275 and Fasc. 4, 179-265. MR0346919 $(49: 11640)$

[27] S. C. Port and R. A. Vitale, Positivity of stable densities, Proc. Amer. Math. Soc. 102 (1988), 1018-1023. MR0934885(89d:60026)

[28] W. E. Pruitt and S. J. Taylor, The potential kernel and hitting probabilities for the general stable process in $R^{N}$, Trans. Amer. Math. Soc. 146 (1969), 299-321. MR 0250372 (40:3611)

[29] K. Sato, Criteria of weak and strong transience for Lévy processes, Probability Theory and Mathematical Statistics, Proc. Seventh Japan-Russia Symp. (ed. S. Watanabe et al.), World Scientific, Singapore, 1996, pp. 438-449. MR1467961 (99c:60157)

[30] K. Sato, Time evolution of Lévy processes, Trends in Probability and Related Analysis, Proc. SAP '96 (ed. N. Kono and N.-R. Shieh), World Scientific, Singapore, 1997, pp. 35-82. MR:1616274 (99f:60135)

[31] K. Sato, Semi-stable processes and their extensions, Trends in Probability and Related Analysis, Proc. SAP'98 (ed. N. Kono and N.-R. Shieh), World Scientific, Singapore, 1999, pp. 129-145. MR.1819201 (2001m:60110)

[32] K. Sato, Lévy Processes and Infinitely Divisible Distributions, Cambridge Univ. Press, Cambridge, 1999. MR 1739520 (2003b:60064)

[33] K. Sato and T. Watanabe, Moments of last exit times for Lévy processes, Ann. Inst. Henri Poincaré, Probab. Statist., 40 (2004), 207-225. MR2044816 (2005c:60054)

[34] K. Sato and T. Watanabe, Last exit times for transient semistable processes, Ann. Inst. Henri Poincaré, Probab. Statist., 41 (2005), 929-951. MR2165258(2006h:60081)

[35] M. J. Sharpe, Supports of convolution semigroups and densities, Probability Measures on Groups and Related Structures, XI (Oberwolfach, 1994), World Scientific, River Edge, NJ, 1995, pp. 364-369. MR.1414946 (98d:60015)

[36] F. Spitzer, Electrostatic capacity, heat flow, and Brownian motion, Zeit. Wahrsch. Verw. Gebiete 3 (1964), 110-121. MR0172343 (30:2562)

[37] J. Takeuchi, Moments of the last exit times, Proc. Japan Acad. 43 (1967), 355-360. MR0222961 (36:6011)

[38] S. J. Taylor, Sample path properties of a transient stable process, J. Math. Mech. 16 (1967), 1229-1246. MR0208684 (34:8493)

[39] A. Tortrat, Le support des lois indéfiniment divisibles dans un groupe Abélien localemnet compact, Math. Z. 197 (1988), 231-250. MR0923491 (89h:60016)

[40] T. Watanabe, The isoperimetric inequality for isotropic unimodal Lévy processes, Zeit. Wahrsch. Verw. Gebiete 63 (1983), 487-499. MR0705619 (84m:60093)

[41] S.J. Wolfe, On the unimodality of multivariate symmetric distribution functions of class $L$, J. Multivariate Analysis, 8 (1978), 141-145. MR0482940(58:2975)

[42] M. Yamazato, Unimodality of infinitely divisible distributions of class L, Ann. Probab. 6 (1978), 523-531. MR0482941 (58:2976)

[43] V. M. Zolotarev, One-Dimensional Stable Distributions, Amer. Math. Soc., Providence, RI. 1986. MR0854867 (87k:60002)

Center for Mathematical Sciences, The University of Aizu, Aizu-Wakamatsu FukuSHIMA, 965-8580 JAPAN

E-mail address: t-watanb@u-aizu.ac.jp 